Research Article

\title{
GuanXinNing Tablet Attenuates Alzheimer's Disease via Improving Gut Microbiota, Host Metabolites, and Neuronal Apoptosis in Rabbits
}

\author{
Feng Zhang $\mathbb{D}^{1}{ }^{1}$ Yanyun $\mathrm{Xu}^{2,3}$ Liye Shen, ${ }^{2,3}$ Junjie Huang, ${ }^{2}$ Songtao $\mathrm{Xu}^{2,3}$ Jiaying $\mathrm{Li}^{4,5}$ \\ Zhichao Sun, ${ }^{4,5}$ Jiangmin He $₫{ }^{6},{ }^{6}$ Minli Chen $\left(\mathbb{0},{ }^{2}\right.$ and Yongming Pan $\mathbb{1}^{2,5}$ \\ ${ }^{1}$ The Second Clinical Medical College, Zhejiang Chinese Medical University, Hangzhou 310053, China \\ ${ }^{2}$ Institute of Comparative Medicine, Experimental Animal Research Center, Zhejiang Chinese Medical University, \\ Hangzhou 310053, China \\ ${ }^{3}$ School of Pharmaceutical Sciences, Zhejiang Chinese Medical University, Hangzhou 310053, China \\ ${ }^{4}$ The First Clinical Medical College, Zhejiang Chinese Medical University, Hangzhou 310053, China \\ ${ }^{5}$ The First Affiliated Hospital of Zhejiang Chinese Medical University, Hangzhou 310006, China \\ ${ }^{6}$ Chiatai Qingchunbao Pharmaceutical Co., Ltd., Hangzhou 310023, China
}

Correspondence should be addressed to Minli Chen; cmli991@zcmu.edu.cn and Yongming Pan; pym@zcmu.edu.cn

Received 30 June 2021; Revised 22 September 2021; Accepted 4 October 2021; Published 28 October 2021

Academic Editor: Weijun Peng

Copyright $\odot 2021$ Feng Zhang et al. This is an open access article distributed under the Creative Commons Attribution License, which permits unrestricted use, distribution, and reproduction in any medium, provided the original work is properly cited.

Based on accumulating evidence, Alzheimer's disease (AD) is related to hypercholesterolemia, gut microbiota, and host metabolites. GuanXinNing Tablet (GXN) is an oral compound preparation composed of two Chinese herbs, Salvia miltiorrhiza Bge. and Ligusticum chuanxiong Hort., both of which exert neuroprotective effects. Nevertheless, the effect of GXN on AD is unknown. In the present study, we investigated whether GXN alters cholesterol, amyloid-beta (A $\beta$ ), gut microbiota, serum metabolites, oxidative stress, neuronal metabolism activities, and apoptosis in an AD model rabbit fed a $2 \%$ cholesterol diet. Our results suggested that the GXN treatment significantly reduced cholesterol levels and A $\beta$ deposition and improved memory and behaviors in AD rabbits. The $16 \mathrm{~S}$ rRNA analysis showed that GXN ameliorated the changes in the gut microbiota, decreased the Firmicutes/Bacteroidetes ratio, and improved the abundances of Akkermansia and dgA-11_gut_group. ${ }^{1} \mathrm{H}-\mathrm{NMR}$ metabolomics found that GXN regulated 12 different serum metabolites, such as low-density lipoprotein (LDL), trimethylamine N-oxide (TMAO), and glutamate (Glu). In addition, the ${ }^{1} \mathrm{H}$-MRS examination showed that GXN remarkably increased $\mathrm{N}$-acetyl aspartate (NAA) and Glu levels while reducing myo-inositol (mI) and choline (Cho) levels in AD rabbits, consequently enhancing neuronal metabolism activities. Furthermore, GXN significantly inhibited oxidative stress and neuronal apoptosis. Taken together, these results indicate that GXN attenuates AD via improving gut microbiota, host metabolites, and neuronal apoptosis.

\section{Introduction}

Alzheimer's disease (AD), mainly manifested by memory and cognitive deterioration, is the most frequent neurodegenerative disease causing dementia in the elderly [1]. In addition to familial $\mathrm{AD}$, sporadic $\mathrm{AD}$ (SAD) accounts for approximately $95 \%$ of the incidence of $\mathrm{AD}$ and is affected by environmental and genetic factors [2]. Currently, approximately 50 million patients have $\mathrm{AD}$ worldwide, and this number is predicted to increase to 131 million by 2050 [3]. Thus, AD has become a growing public health problem [1]. Since the main pathological hallmarks of $\mathrm{AD}$ are $\beta$-amyloid $(\mathrm{A} \beta)$ deposition and tau hyperphosphorylation, increasing research and development strategies of anti-AD drugs focus on these two processes [3]. However, most drugs have failed in the clinical research phase [3], indicating that other pathways are involved in AD. Moreover, currently clinically used N-methyl D-aspartic acid receptor antagonists, 
cholinesterase inhibitors, and other $\mathrm{AD}$ drugs have limited efficacy with varying degrees of adverse reactions, usually increasing the difficulties associated with clinical medications for clinicians and patients $[4,5]$. Therefore, in-depth studies of the mechanism of $\mathrm{AD}$ and its alternative treatment strategies are critical.

In recent years, the gut microbiota has attracted increasing attention due to its critical role in $\mathrm{AD}$. The gut microbiota and its metabolites alter colon permeability and the blood-brain barrier and trigger systemic oxidative stress [6]. Meanwhile, some studies have suggested that gut microbiota disorders affect host nutrition, immunity, and metabolism, and participate in the progression of $\mathrm{AD}$ by the gut-brain axis $[6,7]$. Moreover, patients with $\mathrm{AD}$ exhibit endogenous metabolite disorders and decreased neuronal metabolic activities in the brain [8,9]. Therefore, gut microbiota and host metabolism may be potential targets for AD treatment. Traditional Chinese medicine (TCM) has the characteristics of multiple ingredients, targets, and pathways [10]. As previously reported, some traditional Chinese herbs ameliorate $\mathrm{AD}$ by regulating the gut microbiota and endogenous metabolites, suggesting that TCM may exert a significant effect on modulating gut microbiota and host metabolites $[11,12]$. GuanXinNing Tablet $(\mathrm{GXN})$ is an oral compound preparation composed of two Chinese herbs, Salvia miltiorrhiza Bge. and Ligusticum chuanxiong Hort. [13]. According to our previous liquid chromatographymass spectrometry (LC-MS) analysis, the main constituents of GXN include salvianolic acid B, ferulic acid, and salvianolic acid A [13].

Although GXN exerts multiple pharmacological effects, such as inhibiting platelet aggregation and antioxidant, antiinflammatory, and vascular endothelium protection activities [13], the effects of GXN on AD remain unclear. In addition, studies have found that S. miltiorrhiza Bge., L. chuanxiong Hort., and their active ingredients exert neuroprotective effects $[14,15]$. Furthermore, the bioactive components of Danshen, such as salvianolic acid A and salvianolic acid B, and Chuanxiong's bioactive components, such as ferulic acid, exert their effects by regulating gut microbiota and host metabolites $[16,17]$. Therefore, we speculate that GXN may regulate the gut microbiota and endogenous metabolites and enhance neuronal metabolic activities via the gut-brain axis, thereby ameliorating $\mathrm{AD}$ symptoms.

Diet is considered one of the most critical factors affecting gut microbiota and host metabolites, and high-fat or high-cholesterol diets can cause an imbalance of the gut microbiota and metabolites [18]. In addition, hypercholesterolemia is an important risk factor for $\mathrm{AD}$ [19]. Compared with rodent $\mathrm{AD}$ models (such as APPswe/PS1dE9 double transgenic mice), rabbits produce $A \beta$ peptide lyase, similar to humans $[20,21]$. Moreover, rabbits with $\mathrm{AD}$ induced by a $2 \%$ high-cholesterol diet developed $\mathrm{A} \beta$ deposition in the brain and exhibited more than 10 pathological features similar to those of human patients with $\mathrm{AD}$, such as cognitive decline [19]. Additionally, drug interventions were effective in this rabbit $\mathrm{AD}$ model [22], indicating that $\mathrm{AD}$ rabbits are suitable for SAD research. In the present study, we investigated the effect of GXN on blood lipid levels, behavior, histopathology, gut microbiota, serum metabolites, oxidative stress, neuronal metabolism activities, and apoptosis in this $\mathrm{AD}$ rabbit model to explore its ability to ameliorate $\mathrm{AD}$, reveal the possible mechanism of action, and provide experimental evidence for GXN as a treatment to delay the progression of $\mathrm{AD}$.

\section{Materials and Methods}

2.1. Establishment and Intervention of AD Rabbit Model. Eighteen male white hair and black eyes (WHBE) rabbits aged 2 to 3 months $(2-2.5 \mathrm{~kg}$ ) were provided by the Xin Jian Rabbit Field (Zhejiang, China). The certificate number is SCXK, 2015-0004. All rabbits were raised in the Experimental Animal Research Center, Zhejiang Chinese Medical University (Zhejiang, China), with the certificate number SYXK (ZHE) 2018-0012. Rabbits were housed on a $12 \mathrm{~h}$ light-dark cycle with free access to food and water. All manipulations complied with the Institutional Animal Care and Use Committee (IACUC) of Zhejiang Chinese Medical University (Approval No. 20190930-06). GXN was obtained from Chiatai Qinchunbao Pharmaceutical Co. Ltd. (Hangzhou, China) and has been approved by the China Food and Drug Administration (No. Z20150028). We identified the main constituents of GXN using LC-MS analysis by referring to previous methods [13]. As shown in Figure 1, the most abundant constituents in GXN are salvianolic acid B (peak 9), rosmarinic acid (peak 7), ferulic acid (peak 5), tanshinol (peak 1), chlorogenic acid (peak 3), caffeic acid (peak 4), and salvianolic acid A (peak 10), which are generally consistent with the results reported by Wang et al. [13].

After two weeks of acclimation, the rabbits were randomized into three groups based on weight and total cholesterol level: the NC group (normal chow, $n=6$ ), the AD group ( $2 \%$ cholesterol diet, $n=6)$, and the GXN group ( $2 \%$ cholesterol diet $+\mathrm{GXN}$ intervention, $n=6)$. The rabbits in the GXN group were orally administered $250 \mathrm{mg} / \mathrm{kg}$ GXN daily for 12 weeks. This dose in the study was determined to be optimal based on our preliminary experiments. The experimental design process is shown in Figure 2. After 12 weeks of treatment, all rabbits were dissected after euthanasia.

2.2. Memory Behavior Test. Twelve weeks after administration, the rabbits fasted for 12 hours, and then the memory and behavioral abilities were evaluated using previously reported methods [23]. Drinking water and feed were placed at a fixed site in the $5.4 \mathrm{~m}^{2}$ rectangular activity area. Training occurred on the day before the test. During the trial, the feed and drinking water were removed from the fixed point, and SMART video tracking system software (v3.0, Panlab, Spain) was used to record the distances traveled by and times to reach the fixed point within 5 minutes of all rabbits.

2.3. Detection of Blood Biochemical Parameters. Each rabbit fasted for 12 hours, and blood biochemical parameters were detected in the 12th week using the corresponding kits 


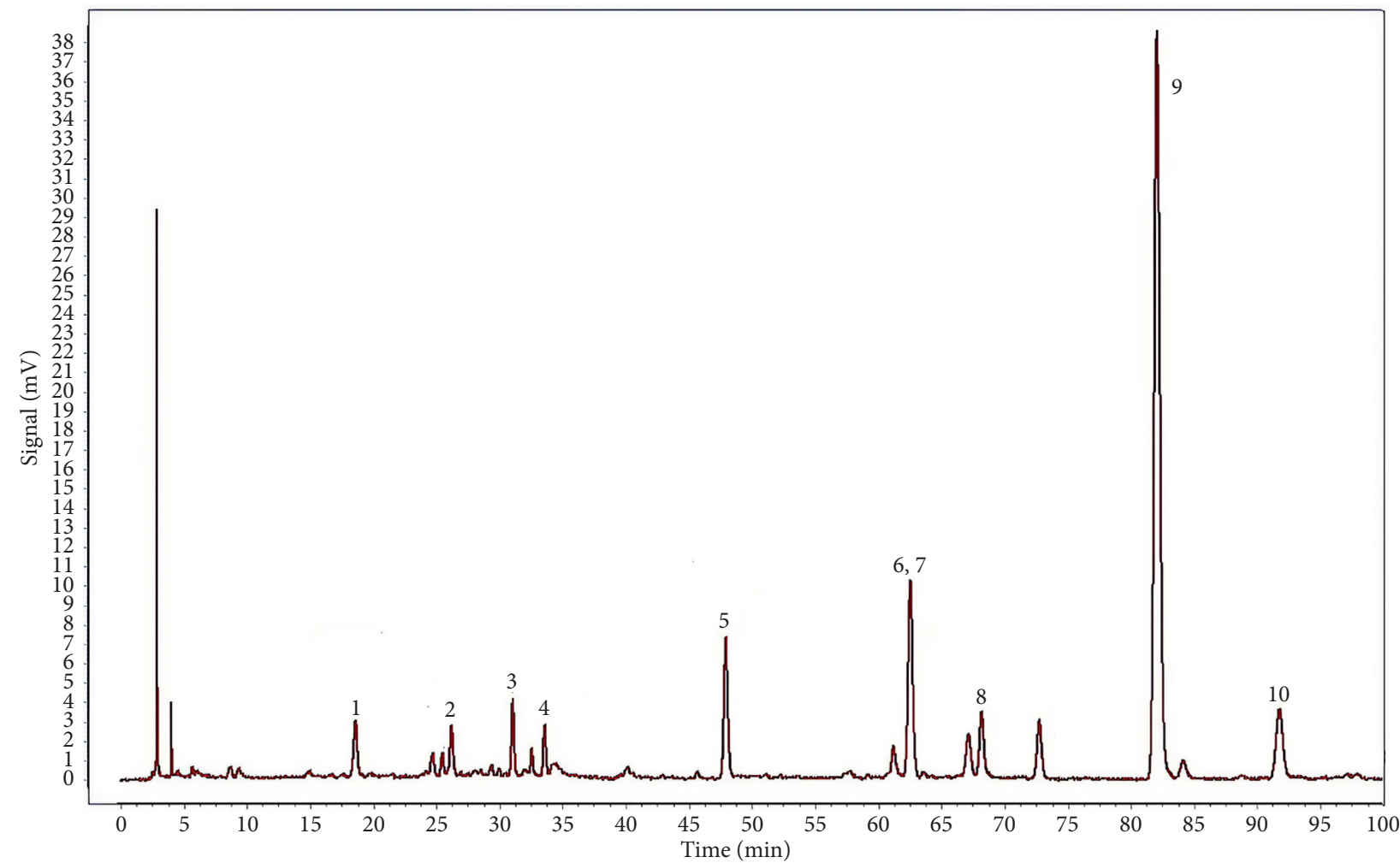

Figure 1: LC-MS chromatogram of GXN: (1) tanshinol, (2) protocatechualdehyde, (3) chlorogenic acid, (4) caffeic acid, (5) ferulic acid, (6) senkyunolide I, (7) rosmarinic acid, (8) isosalvianolic acid A, (9) salvianolic acid B, and (10) salvianolic acid A.

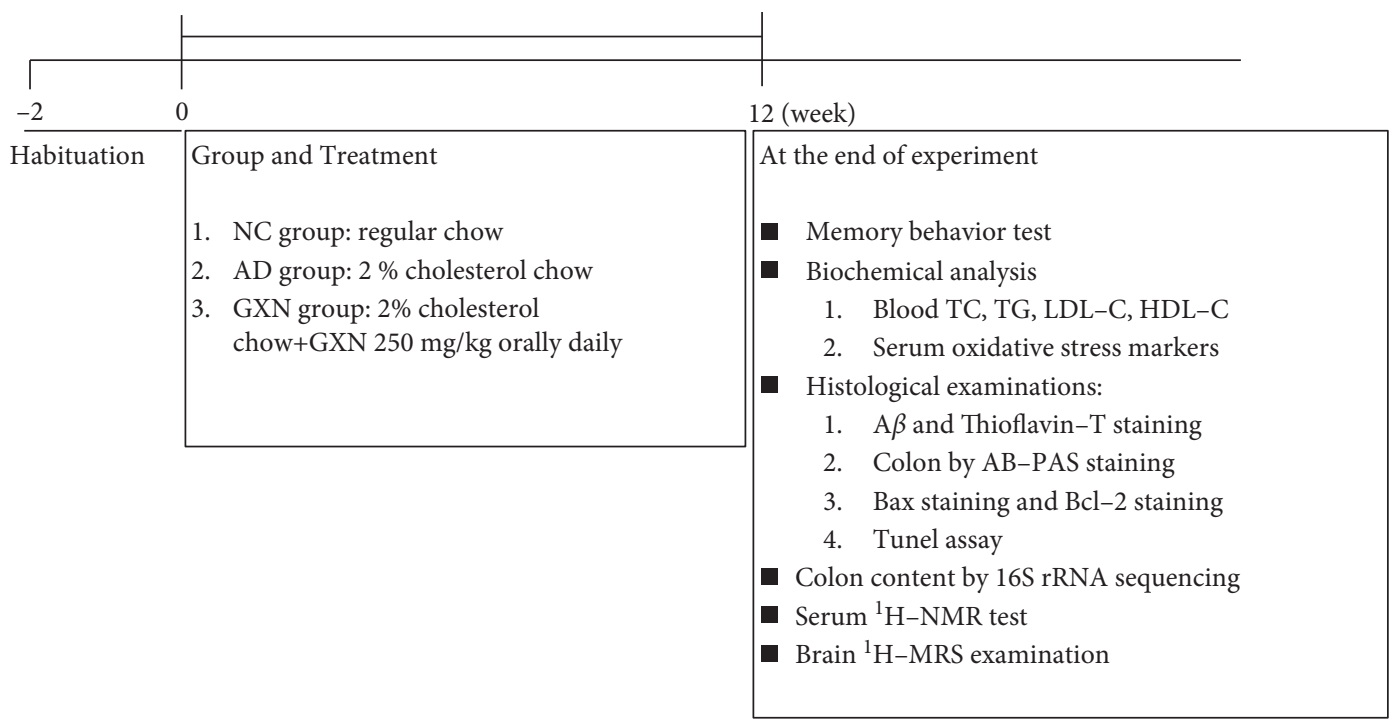

Figure 2: Flow chart of the study.

(Ningbo Medical System Biotechnology Co. Ltd., China). Blood was obtained via the middle auricular artery. Plasma total cholesterol (TC), triglycerides (TG), high-density lipoprotein cholesterol (HDL-C), and low-density lipoprotein cholesterol (LDL-C) levels were detected using a biochemical analyzer (7020, ITACHI, Japan). In addition, serum levels of oxidative stress markers were measured according to the instructions of the superoxide dismutase
(SOD) and malondialdehyde (MDA) kits (Jiancheng Bioengineering Institute, Nanjing, China).

2.4. Histopathological Examinations. After an intravenous injection of pentobarbital sodium for anesthesia, the rabbits were perfused transcardially with $300 \mathrm{ml}$ of $4^{\circ} \mathrm{C}$ PBS. The brain tissues and colon tissues were removed, sliced, and 
fixed with formalin for $24 \mathrm{~h}$. After fixation, sections were dehydrated with a gradient of ethanol solutions, embedded, and sliced. A Hamamatsu Skeleton Scanner (Nanozoomer S210, Hamamatsu, Japan) was used to scan all sections.

$\mathrm{A} \beta$ immunohistochemical staining was performed to examine $\mathrm{A} \beta$ deposition. Bax staining and $\mathrm{Bcl}-2$ staining were performed to evaluate apoptosis levels. Briefly, the paraffin sections were dewaxed in water, and peroxidase activity was blocked with $3 \% \mathrm{H}_{2} \mathrm{O}_{2}$. Then, the sections were incubated with $\beta$-amyloid (1:100), $\operatorname{Bax}(1: 200)$, and Bcl-2 (1: 200) antibodies (Santa Cruz Biotechnology, USA) at $4^{\circ} \mathrm{C}$ overnight. Subsequently, all sections were incubated with the secondary antibody (IgG 1:1000) for 1 hour, stained with $\mathrm{DAB}$, and counterstained with hematoxylin. The positive reaction was specifically stained pale yellow or brownish-yellow.

Thioflavin-T staining was conducted to detect the $\mathrm{A} \beta$ content in the brain. Briefly, slides were dewaxed, hydrated in ethanol, and incubated with Mayer's hematoxylin for five minutes. Then, the sections were washed with DW two times and stained with 1\% thioflavin-T (Dalian Meilun Biological Technology Co. Ltd., China) for $10 \mathrm{~min}$. After three washes with DW, fluorescence was excited, and the sections were scanned.

IPP 6.0 software was used to analyze the percentages of $\mathrm{A} \beta$-positive and thioflavin-T-positive areas in the entire visual field of the frontal cortex and hippocampus.

Alcian blue and periodic acid-S chiff (AB-PAS) staining were performed to observe colonic epithelial barrier function. The mucus-secreting goblet cells per colonic crypt were stained blue. Briefly, paraffin sections were dewaxed in water, dyed with Alcian blue for $20 \mathrm{~min}$, oxidized in periodic acid for $5 \mathrm{~min}$, and depigmented with Schiff reagent for $20 \mathrm{~min}$. Then, the sections were rinsed, counterstained with hematoxylin, placed in an acidic differentiation solution to induce differentiation, and counterstained blue using Scotts bluing reagent. The ABPAS positive cells in each crypt from each rabbit in the three groups were counted in five different areas per section. All counts were performed by two independent observers.

2.5. $16 S$ rRNA Gene Sequencing and Data Analysis. Twelve weeks after administration, samples of the colon contents were obtained from all rabbits and stored at $-80^{\circ} \mathrm{C}$, with five samples per group. Total DNA was extracted from the colon contents, and the extraction quality was assessed using agarose gel electrophoresis. Subsequently, the amplicons of the V3-V4 variable region of the $16 \mathrm{~S}$ rRNA were amplified with specific primers 341F (5'-CCTACGGGNGGCWGCAG-3') and 805R $\left(5^{\prime}-\right.$ GACTACHVGGGTATCTAATCC- $\left.3^{\prime}\right)$. $16 \mathrm{~S}$ rRNA sequencing library was constructed, and $2 \times 250$ bp pairedend sequencing was performed using a NovaSeq sequencer with the assistance of Hangzhou Lianchuan Biotechnology Co. Ltd. Cutdapt 1.9 software was used to filter low-quality data from the raw sequencing data to ensure the accuracy of the subsequent analysis. FLASH
1.2.8 software was used for double-end splicing, and the double-end data were merged into a long amplicon fragment. Then, the QIIME2 DADA2 plug-in was used for sequence quality control and feature table construction, the QIIME 2 feature-classifier (2019.7) was used for sequence alignment, and the species annotation databases SILVA (release 132) and NT-16S (2019.04.05) were used to perform annotations. Alpha diversity (Chaol and Shannon) and beta diversity were analyzed. The relative abundances of phyla and genera were compared using Welch's $t$-test in STAMP software. The Kruskal-Wallis test was used to statistically analyze the microbial components, and then the linear discriminant effect size (LEfSe) analysis was performed with the unpaired Wilcoxon test (available from https://huttenhower.sph.harvard.edu/galaxy/).

2.6. ${ }^{1} \mathrm{H}-\mathrm{NMR}$ and Data Analysis. The thawed serum samples $(450 \mu \mathrm{L})$ and D2O $(350 \mu \mathrm{L})$ were added to an EP tube. The mixture was vortex mixed thoroughly and centrifuged at $13,000 \mathrm{rpm}$ for $20 \mathrm{~min}$ at $4^{\circ} \mathrm{C}$. The supernatant $(600 \mu \mathrm{l})$ was transferred to an NMR tube with a diameter of $5 \mathrm{~mm}$, and NMR spectra were recorded by a Bruker Avance II NMR spectrometer (Bruker BioSpin, Germany). ${ }^{1} \mathrm{H}$-NMR free induction decay (FID) signals were input into Chenomx NMR Suite version 7.7 software (Chenomx, Edmonton, Canada), and automated Fourier transform, phase, and baseline corrections were performed. The ${ }^{1} \mathrm{H}-\mathrm{NMR}$ map phase and baseline were adjusted using MestReNova 14 (Mestrelab Research, Santiago deCompostella, Spain). The DSS-d6 peak $(0.0 \mathrm{ppm})$ was regarded as the internal standard of all chemical shifts; deconvolution was conducted; and all peak shapes were adjusted. According to the relevant information of the signal in the ${ }^{1} \mathrm{H}$-NMR spectrum, the concentration and peak area of DSS-d6 were used as the standards to normalize the integrated data. Then, we saved the resulting data in an Excel file for further analysis. SIMCA-P14.1 (Umetrics, Umeå, Sweden) was applied to centralize and normalize the integral data. Partial least squares-discriminant analysis (PLS-DA) was performed to observe the dispersion between groups. A permutation test (200 times) was conducted to verify the effectiveness of the PLS-DA model, and orthogonal partial least squares-discriminant analysis (OPLS-DA) was conducted to identify the differentially produced metabolites between samples.

2.7. Brain ${ }^{1} H$-MRS Examination. After 12 weeks of administration, proton magnetic resonance spectroscopy $\left({ }^{1} \mathrm{H}\right.$ MRS) scanning was performed on a 3.0T MRI scanner. Point resolved spectroscopy (PRESS) was used in ${ }^{1} \mathrm{H}$-MRS scanning. LC Model (Provencher SW, version 6.3) was used to analyze the spectra. The metabolites to be observed in the rabbit brain included the changes in $\mathrm{N}$-acetylaspartic acid (NAA), glutamate complex (Glx), choline (Cho), and myoinositol (mI) levels, all of which were standardized to creatine $(\mathrm{Cr})$ levels. All operations were performed according to 


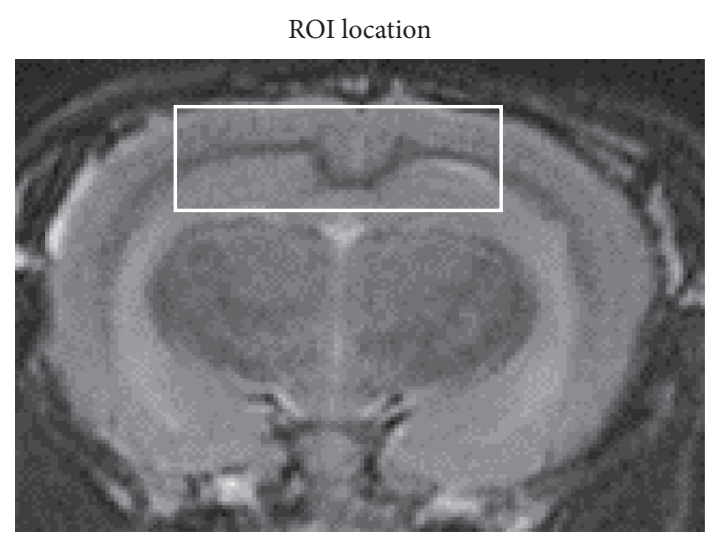

(a)

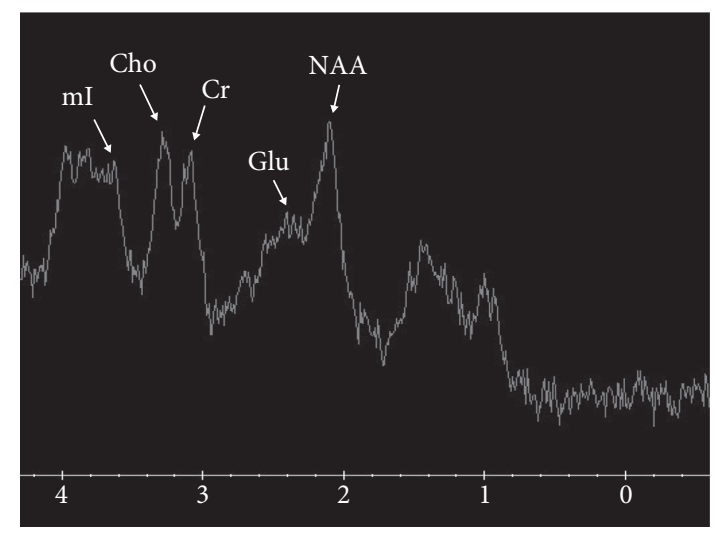

(b)
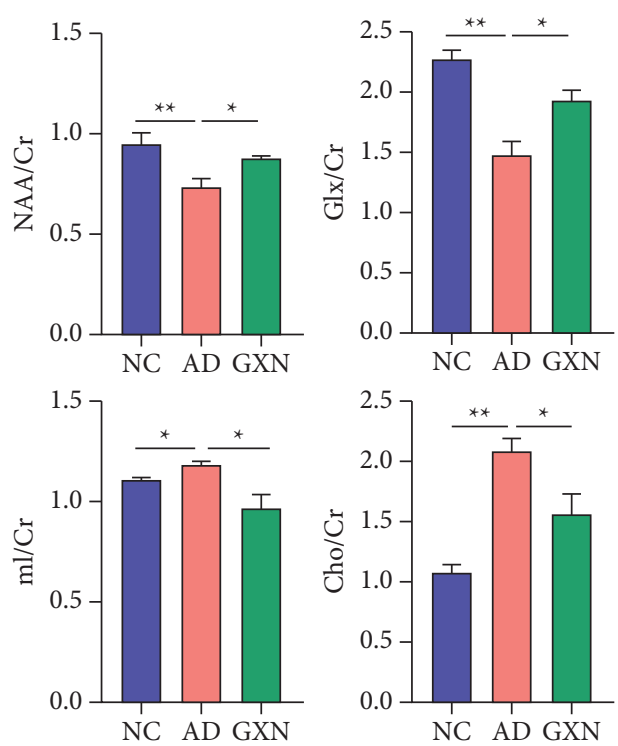

(c)

FIGURE 3: GXN improved rabbit neuronal metabolism activities: (a) the white box indicates the ROI in the brain, (b) the metabolites in ${ }^{1} \mathrm{H}$ MRS spectra were identified, and (c) changes in metabolites in each group $(n=6)$.

previous research methods $[24,25]$. The frontal cortex and hippocampus in the brain were the regions of interest (ROI; Figure 3(a)).

2.8. TUNEL Staining of Brain Tissues. We determined neuronal apoptosis by conducting a TUNEL assay according to the instructions provided with the corresponding kits (Baori Medical Biotechnology Co. Ltd., China). Images of six randomly selected visual fields of the cortex and the hippocampus (three fields for each region) were captured. TUNEL-positive cells were stained green, and nuclei were stained blue. The number of positive cells and the total number of neurons per field were counted, and the apoptosis index (AI; $\mathrm{AI}=$ number of positive cells/total number of neurons) was calculated.

2.9. Statistical Analysis. Data are presented as means \pm SEM. GraphPad Prism 8.0 software (GraphPad Software, Inc., La Jolla, CA, USA) was applied for statistical analyses using one-way ANOVA with Tukey's post hoc test $\left({ }^{*} P<0.05\right.$ and ${ }^{* *} P<0.01$ versus the AD model group). Pearson's correlation coefficient $(R)$ was calculated to analyze correlations. $P<0.05$ was considered statistically significant.

\section{Results}

3.1. GXN Reduced Blood Lipid Levels, Improved Memory and Behaviors, and Alleviated $A \beta$ Pathology in the Brains of $A D$ Rabbits. As shown in Figure 4(a), the GXN group showed $31.6 \%$ and $37.8 \%$ reductions in plasma levels of TC and LDL-C compared to the AD group, respectively $(P<0.01)$. In addition, the body weight of the three groups did not change significantly $(P>0.05)$. Furthermore, the memory and behavioral tests suggested that the distance traveled and time to reach a fixed point in the AD group were significantly prolonged compared with those in the NC group $(P<0.01)$. In contrast, GXN treatment significantly reduced the distance traveled and time by $69.6 \%$ and $57.4 \%$, respectively $(P<0.01$; Figures $4(\mathrm{~b})$ and $4(\mathrm{c}))$. In addition, $\mathrm{A} \beta$-staining showed 

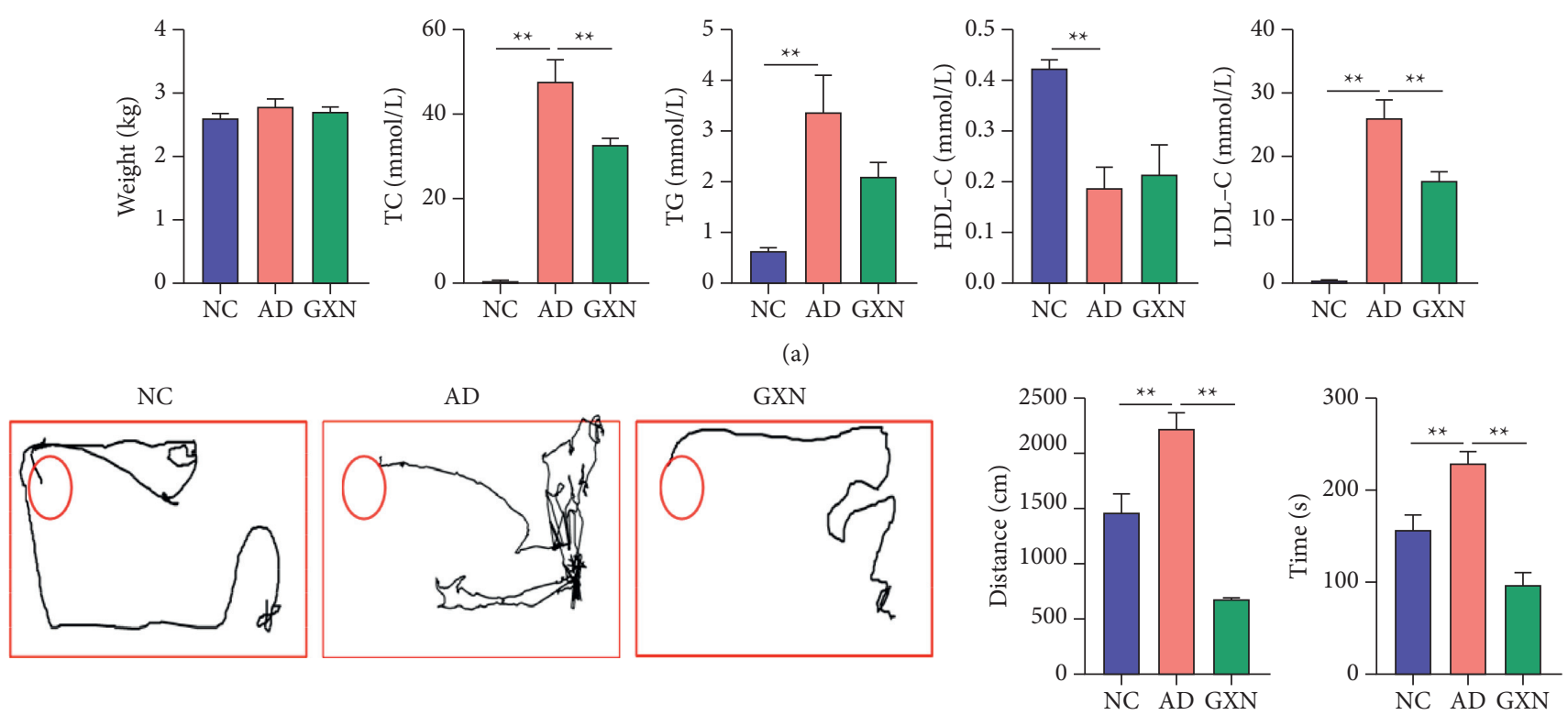

(b)
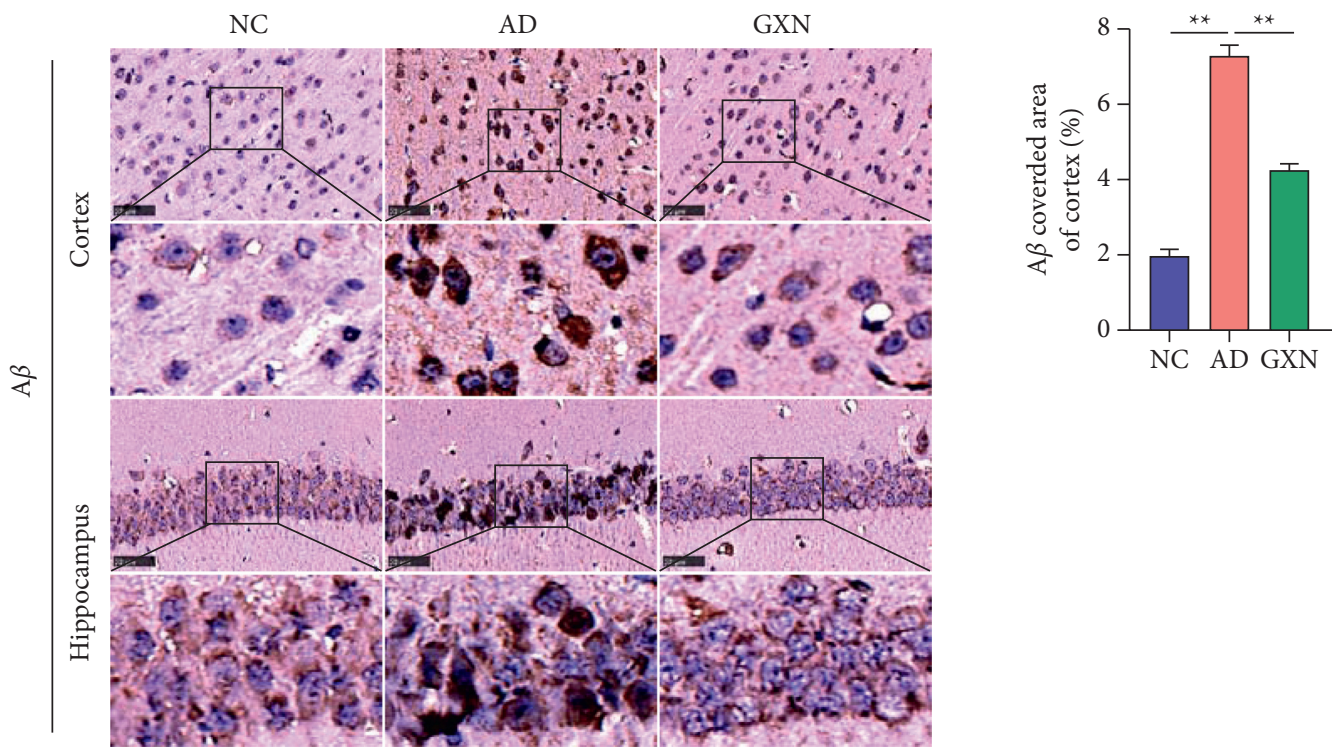

(c)

(d)

(e)

Figure 4: Continued.

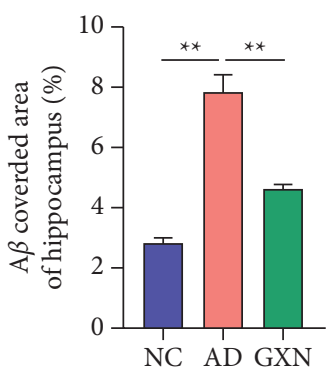




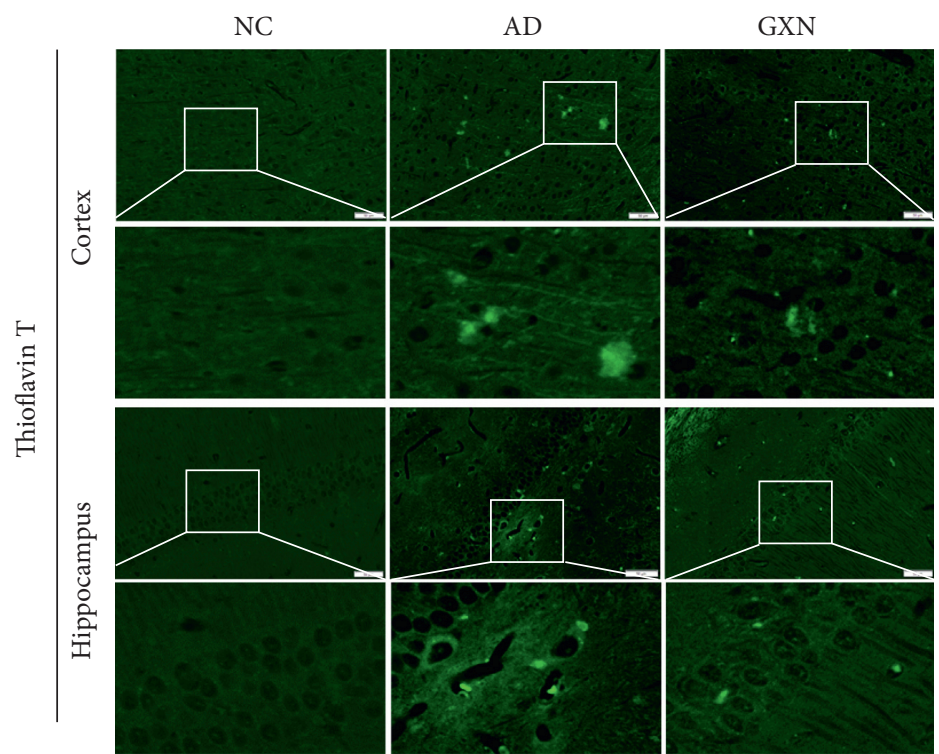

(f)

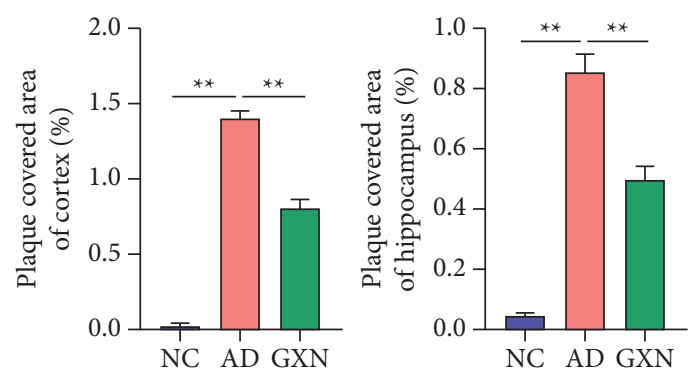

(g)

FIGURE 4: GXN reduced blood lipid levels, improved memory and behaviors, and alleviated $\mathrm{A} \beta$ deposition in the brains of $\mathrm{AD}$ rabbits. (a) The effects of GXN on body weight and TC, TG, HDL-C, and LDL-C levels in AD rabbits. (b) Representative traces of the path traveled by each group in the memory and behavior test. (c) Quantitative analysis of the distance traveled and time to reach a fixed point of each group. $(\mathrm{d}, \mathrm{f})$ Representative images of $\mathrm{A} \beta$ and thioflavin-T staining in each group. Scale bar $=50 \mathrm{~mm}$. White or black boxes indicate the region shown at higher magnification below each panel. $(\mathrm{e}, \mathrm{g})$ Quantitative analysis of $\mathrm{A} \beta$ levels and plaque-covered area in each group. $n=6$.

significantly decreased $\mathrm{A} \beta$ expression in both the cortex and hippocampus of the GXN group relative to the AD group $(P<0.01$; Figures $4(\mathrm{~d})$ and $4(\mathrm{e}))$. Moreover, thioflavin-T staining showed that the GXN intervention significantly reduced the plaque-covered areas in both the cortex and hippocampus compared to the $\mathrm{AD}$ group $(P<0.01$; Figures $4(\mathrm{f})$ and $4(\mathrm{~g}))$.

\subsection{GXN Improved Colon Barrier Permeability and the Di-} versity of Gut Microbiota in AD Rabbits. As a dynamic semipermeable barrier, the gut mucus layer is a vital component of the colon barrier. In the NC group, the epithelial cells and goblet cells in the colonic mucosal layer exhibited a normal morphology, and abundant mucus secretion on the mucosal surface was observed, whereas in the $\mathrm{AD}$ group these cells showed variable degrees of atrophy and mucus secretion decreased (Figure 5(a)). Compared with the AD group, the epithelial cells and goblet cells at various levels of recovery were observed in the GXN group, and mucus secretion also increased. Quantitatively, the GXN intervention significantly increased the number of AB-PAS-positive cells per crypt $(P<0.05$; Figure $5(\mathrm{~b}))$. In addition, $\alpha$-diversity is a composite indicator used to assess species evenness and richness in a single community sample. The Chaol index mainly reflects the abundance of species in the sample, and the Shannon index mainly reflects the diversity of species in the community. Compared with the NC group, the Chaol index in the AD group was slightly increased (Figure 5(c); $P>0.05$ ), while the Shannon index was significantly increased (Figure $5(\mathrm{~d}) ; P<0.05$ ). Nevertheless, in the GXN group, the Chao1 index was slightly decreased compared to that in the AD group $(P>0.05)$, while the Shannon index was significantly decreased $(P<0.05) . \quad \beta$-Diversity reflects changes in the gut microbiota structure among different samples. As shown in Figure 5(e), principal coordinate analysis (PCoA; $P=0.004$ ) suggested that the AD group significantly deviated from the NC group, and the GXN group was nearer to the NC group than the AD group.

3.3. GXN Improved the Structure of the Gut Microbiota in $A D$ Rabbits. Eleven major phyla were identified in the gut microbiota of WHBE rabbit colon contents, including Firmicutes, Bacteroidetes, and Verrucomicrobia (Figure 6(a)). Compared with the AD group, the GXN intervention significantly decreased the Firmicutes/Bacteroidetes ratio and the abundance of Firmicutes $(P<0.05$ and $P<0.01$; Figures 6(b) and 6(c)) and significantly increased the abundance of Verrucomicrobia $(P<0.05$; Figure $6(\mathrm{c}))$, indicating that GXN ameliorated gut microbiota disorders.

The classification level was further refined to the genus level, and the top 20 genera are listed in Figure 6(d). In the comparison of the $\mathrm{AD}$ and $\mathrm{NC}$ groups, 10 bacterial genera were significantly changed $(P<0.05$ and $P<0.01$; Figure 6(e)), including Akkermansia, Firmicutes_unclassified, and dgA-11_gut_group. Meanwhile, in the comparison of the GXN and AD groups, the abundance of five bacterial genera (Akkermansia, dgA-11_gut_group, Ruminiclostridium_5, Intestinimonas, and Moryella) was significantly altered $(P<0.05)$. Furthermore, an LEfSe analysis was conducted using the Galaxy version 1.0.0 online analysis tool to further determine the bacteria targeted by GXN. The biomarkers of the gut microbiota on the evolutionary branch 

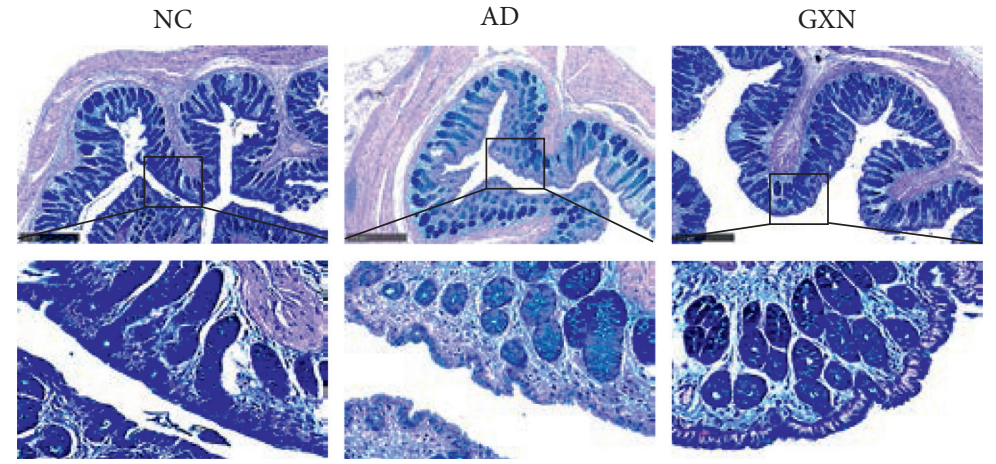

(a)

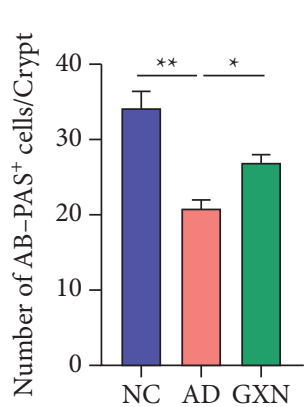

(b)

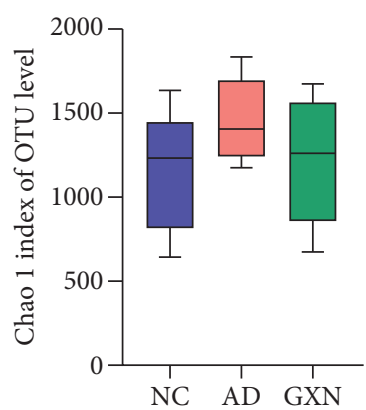

(c)

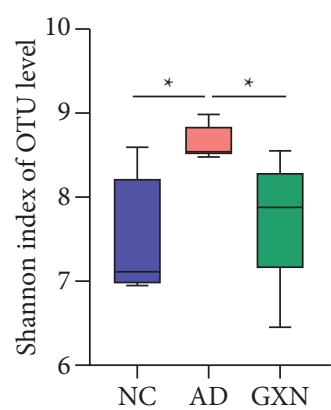

(d)

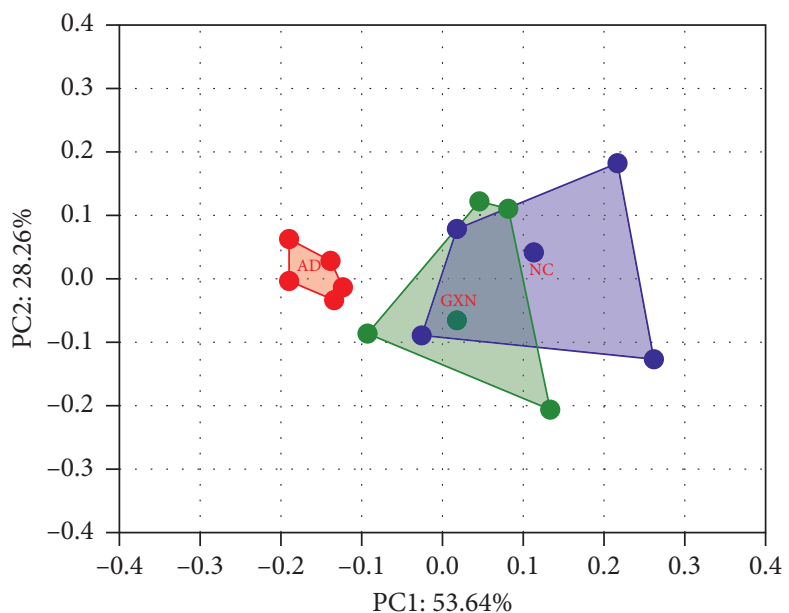

(e)

FIGURE 5: GXN improved colon barrier permeability and the diversity of gut microbiota in AD rabbits. (a) Typical images of AB-PAS staining in the colon tissue. Scale bar $=500 \mathrm{~mm}$. Black boxes indicate the areas shown at higher magnification. (b) Quantitative analysis of the number of AB-PAS-positive cells per crypt in each group. Chaol (c) and Shannon (d) indices of $\alpha$-diversity were assessed. (e) Principal coordinate analysis (PCoA) of the $\beta$-diversity index. $n=5$.

at each level of classification were detected. The results further confirmed that Akkermansia and dgA-11_gut_group were significantly affected in the $\mathrm{AD}$ rabbit, and the GXN intervention significantly regulated the abundance of these bacteria (Figure 6(f)), suggesting that Akkermansia and $d g A$ 11_gut_group may be the target bacterial genera of the antiAD effects of GXN.

Pearson's correlation analysis was conducted to assess the correlations between representative gut microbiota, blood lipid levels, $\mathrm{A} \beta$, and neuronal metabolism activities in $\mathrm{AD}$ rabbits. As shown in Figure 6(g), TC, LDL-C, and $\mathrm{A} \beta$ levels in the hippocampus and cortex, and $\mathrm{mI}$ and Cho levels were positively associated with the dgA-11_gut_group $(P<0.05$ and $P<0.01)$, while Glx and NAA levels showed a negative correlation $(P<0.01)$. In addition, Akkermansia was negatively correlated with TC, LDL-C, $\mathrm{A} \beta$, and $\mathrm{mI}$ levels $(P<0.05)$.

3.4. GXN Altered Serum Metabolite Levels in AD Rabbits. Twenty-four endogenous metabolites were identified by analyzing the characteristics of the spectra and combining 

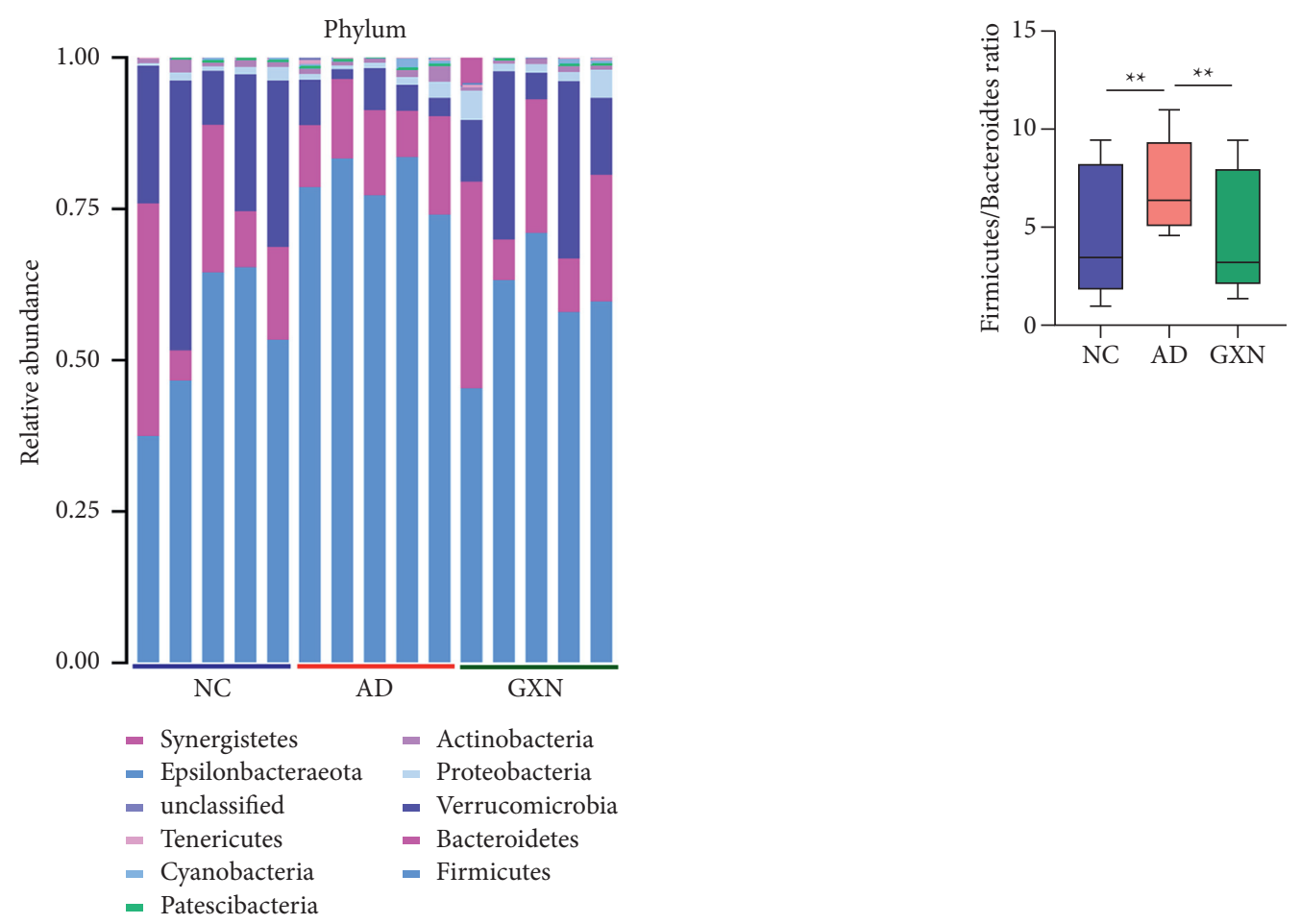

(a)

(b)
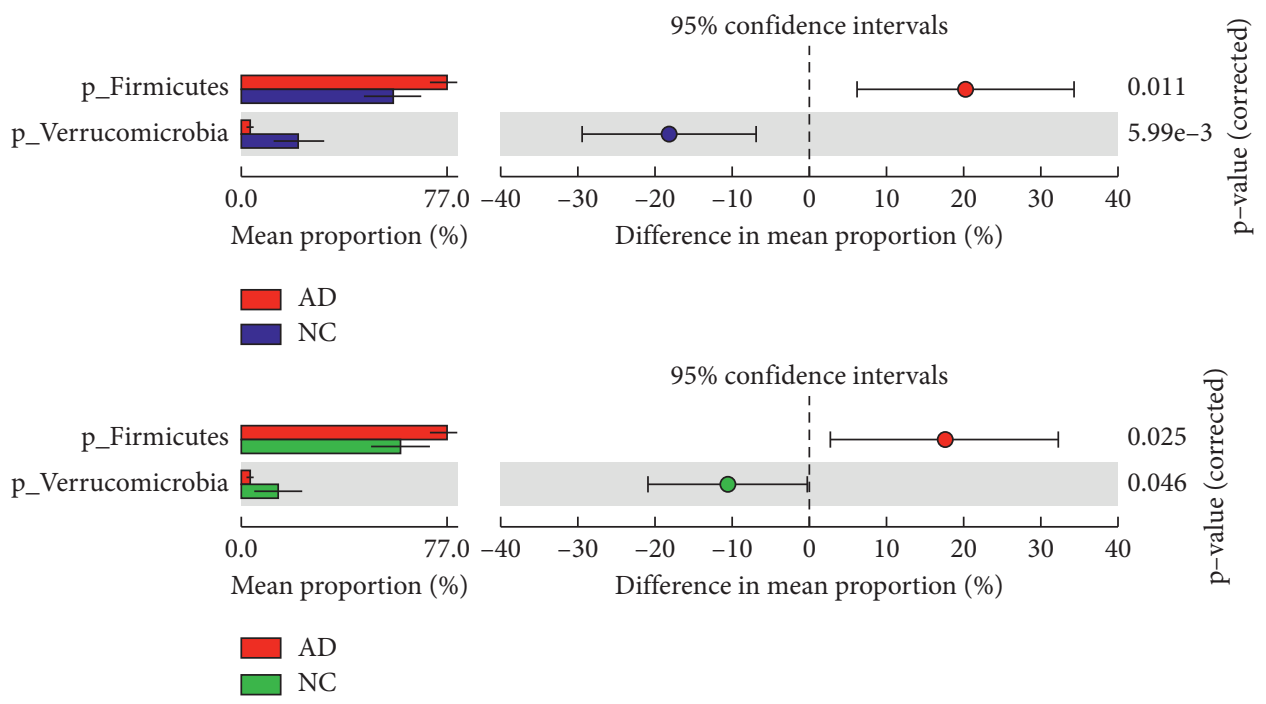

(c)

Figure 6: Continued. 


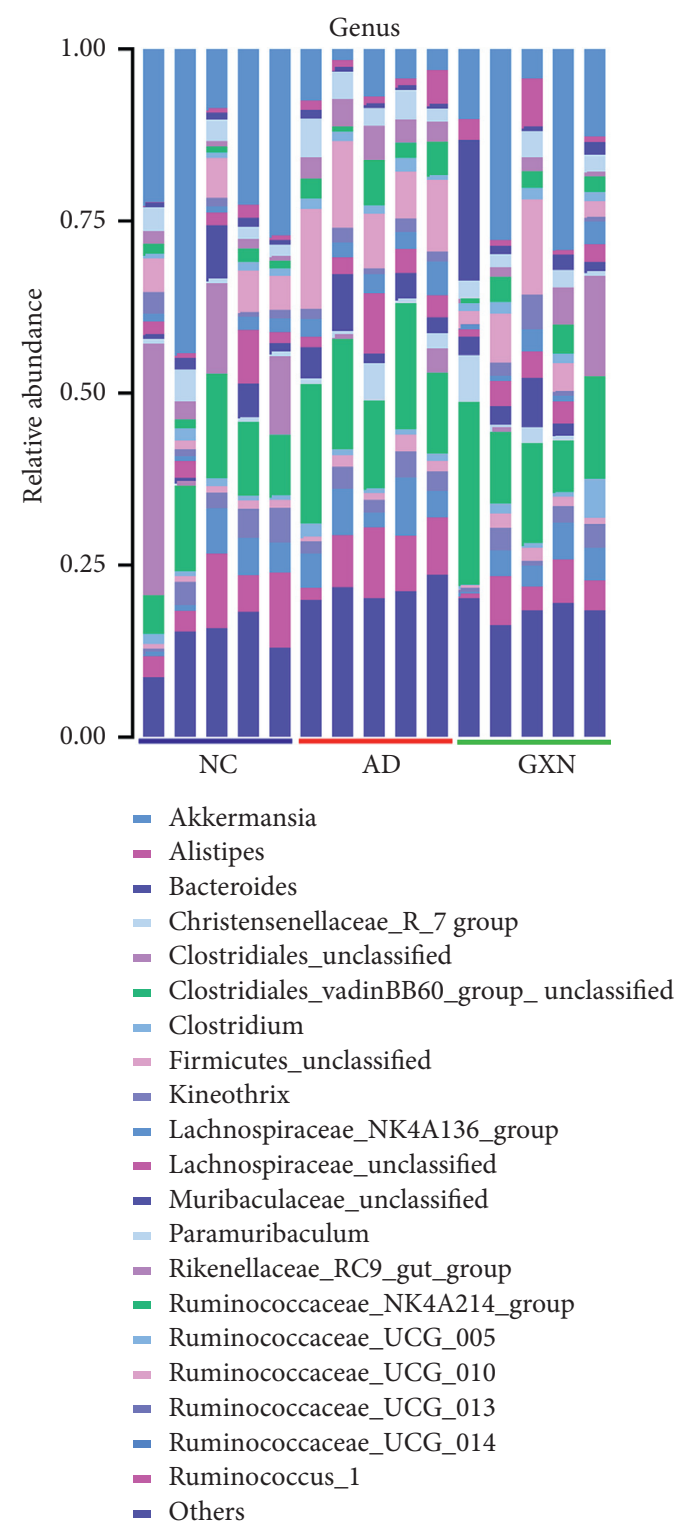

(d)

FIgURE 6: Continued. 

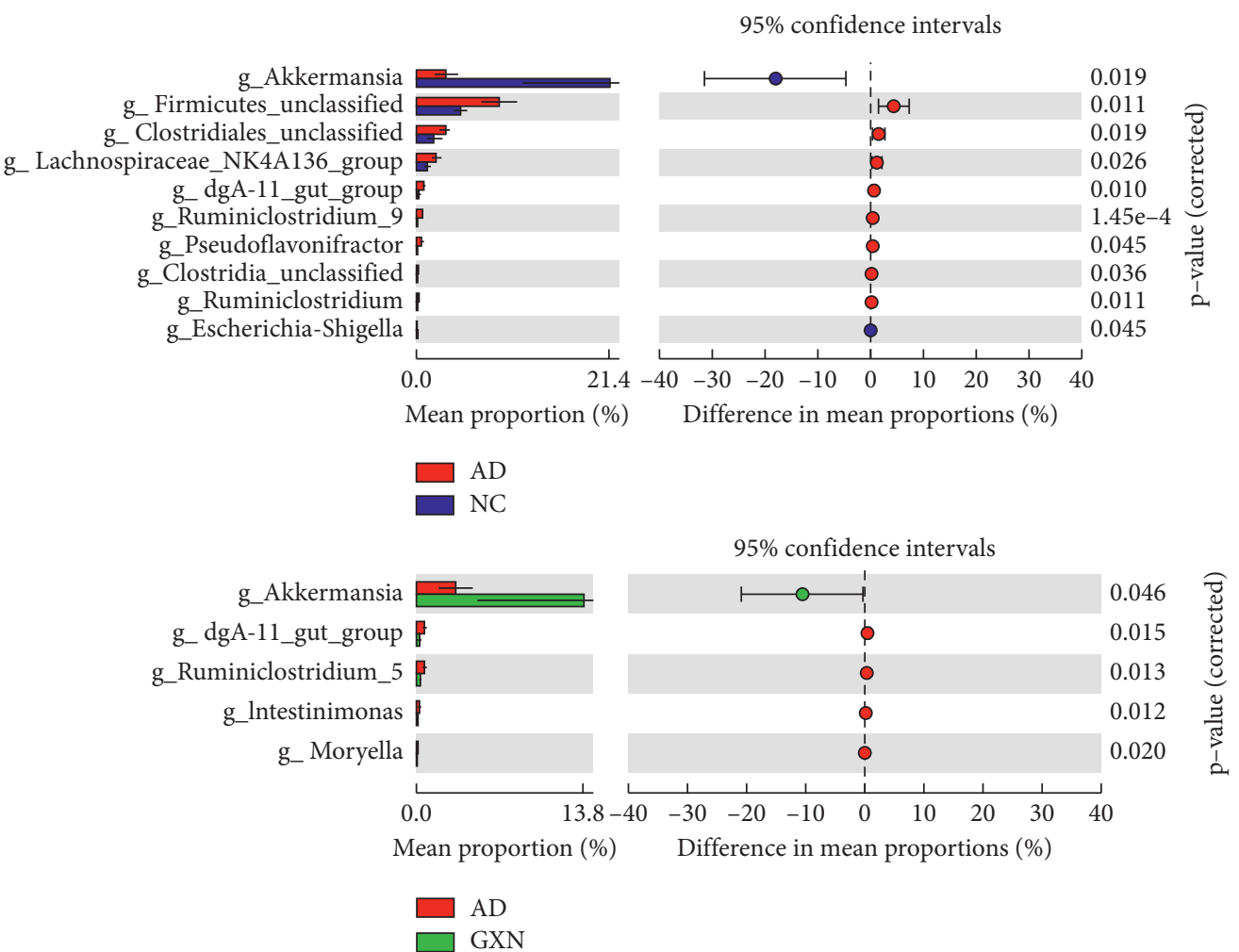

(e)
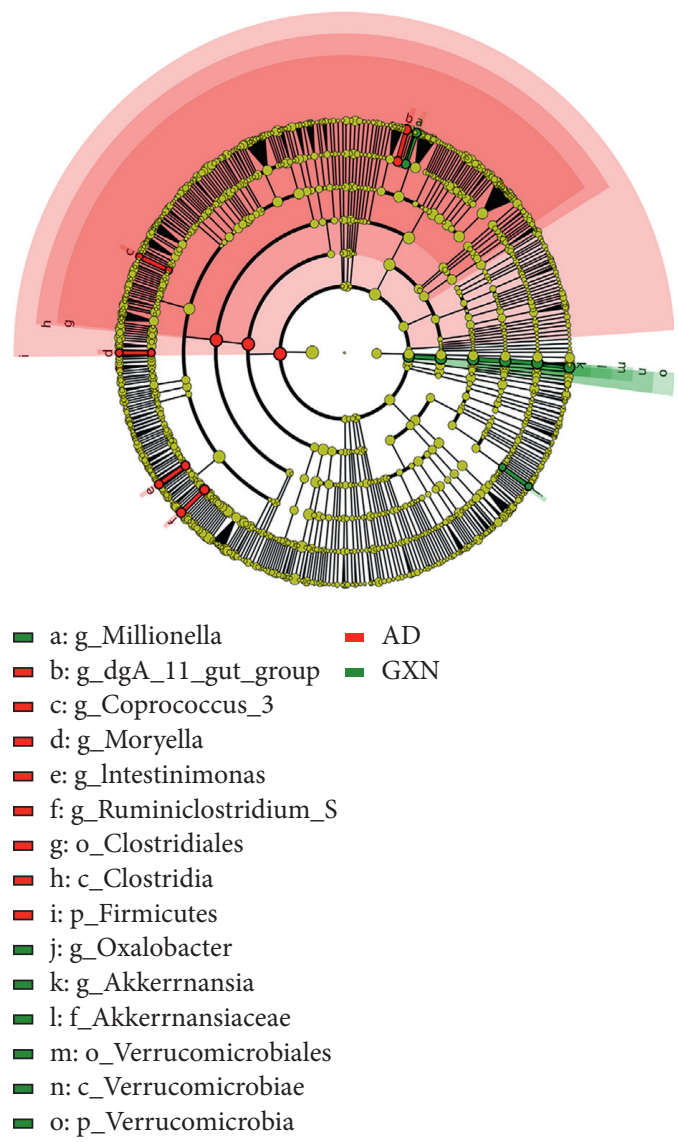

Figure 6: Continued. 


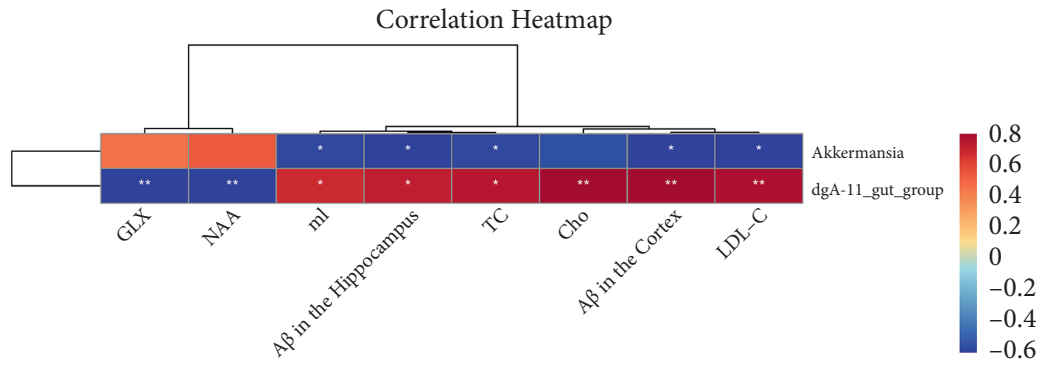

(g)

FIgURE 6: GXN improved the structure of the gut microbiota in AD rabbits: (a) and (d) relative abundance at the phylum and genus levels; (b) the Firmicutes/Bacteroidetes ratio; (c) and (e) changes in specific phyla and genera; (f) evolutionary branch diagrams comparing the gut microbiota in the AD and GXN groups; and (g) heatmap of Pearson's correlation coefficients between representative components of the gut microbiota, blood lipids, $\mathrm{A} \beta$, and neuronal metabolism activities. $n=5$. Red and blue indicate positive correlations and negative correlations, respectively.

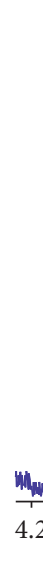

$\mid$

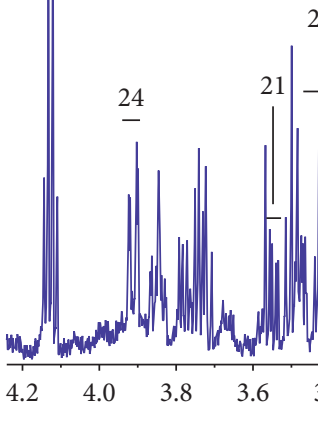<smiles>C[Si]12CCC(CC1)C2</smiles><smiles>CC(C)(C)C</smiles><smiles>C1CCC2CCCCC2C1</smiles>

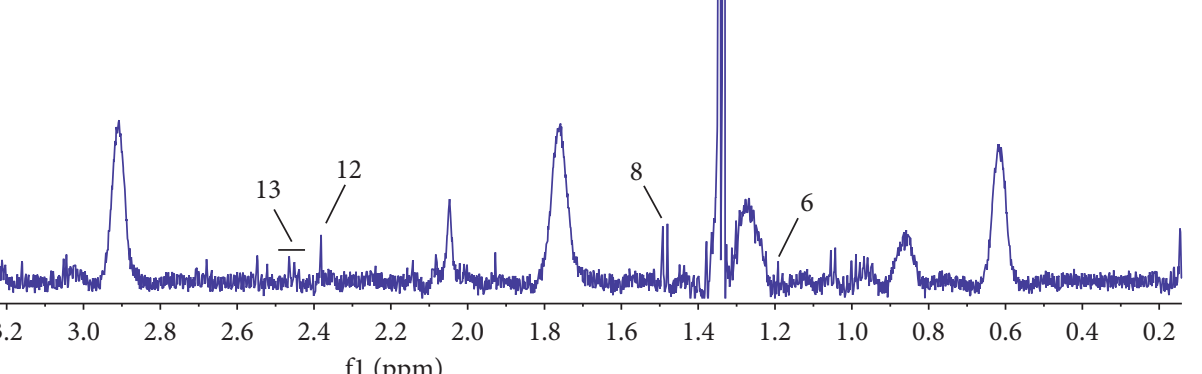

(a)

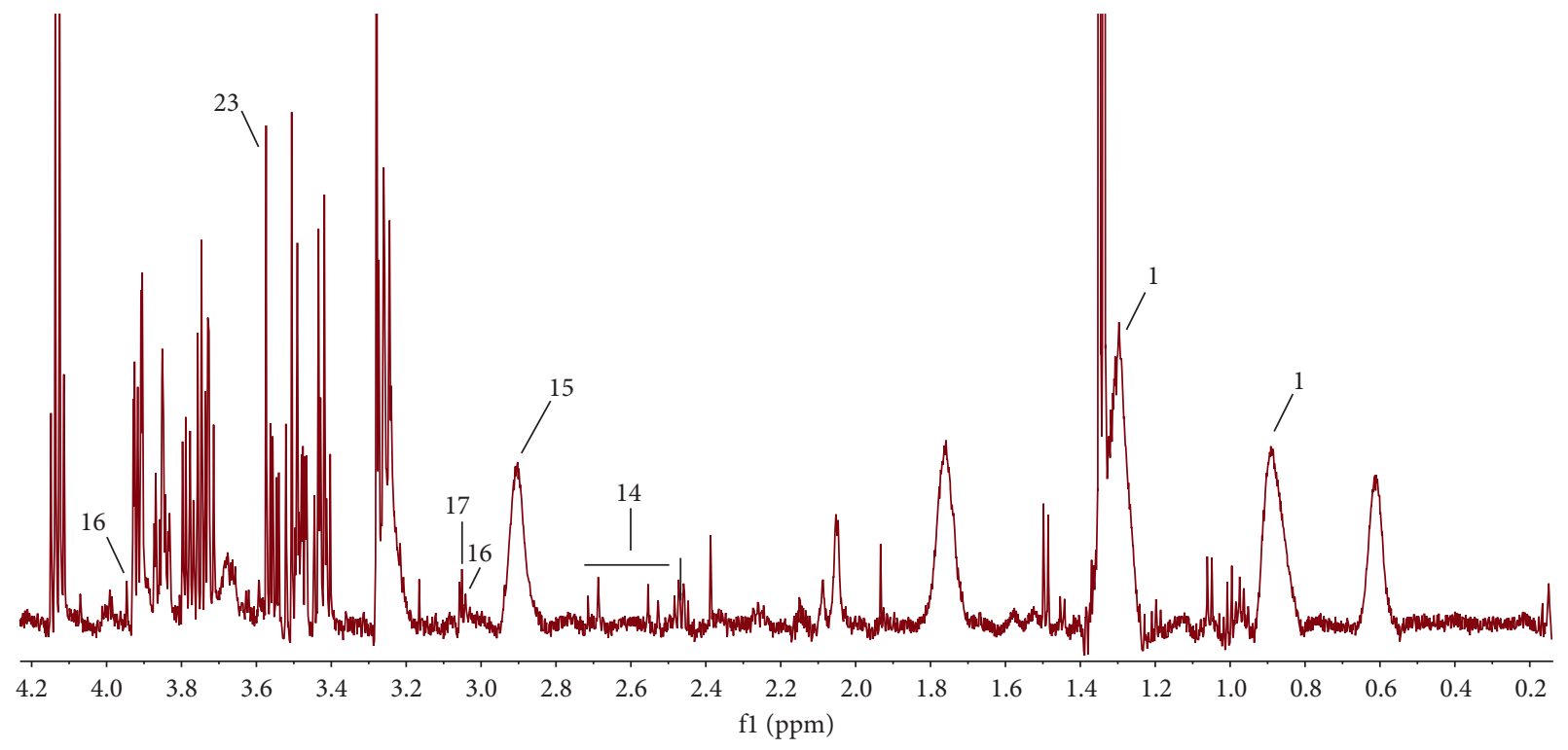

(b)

Figure 7: Continued. 


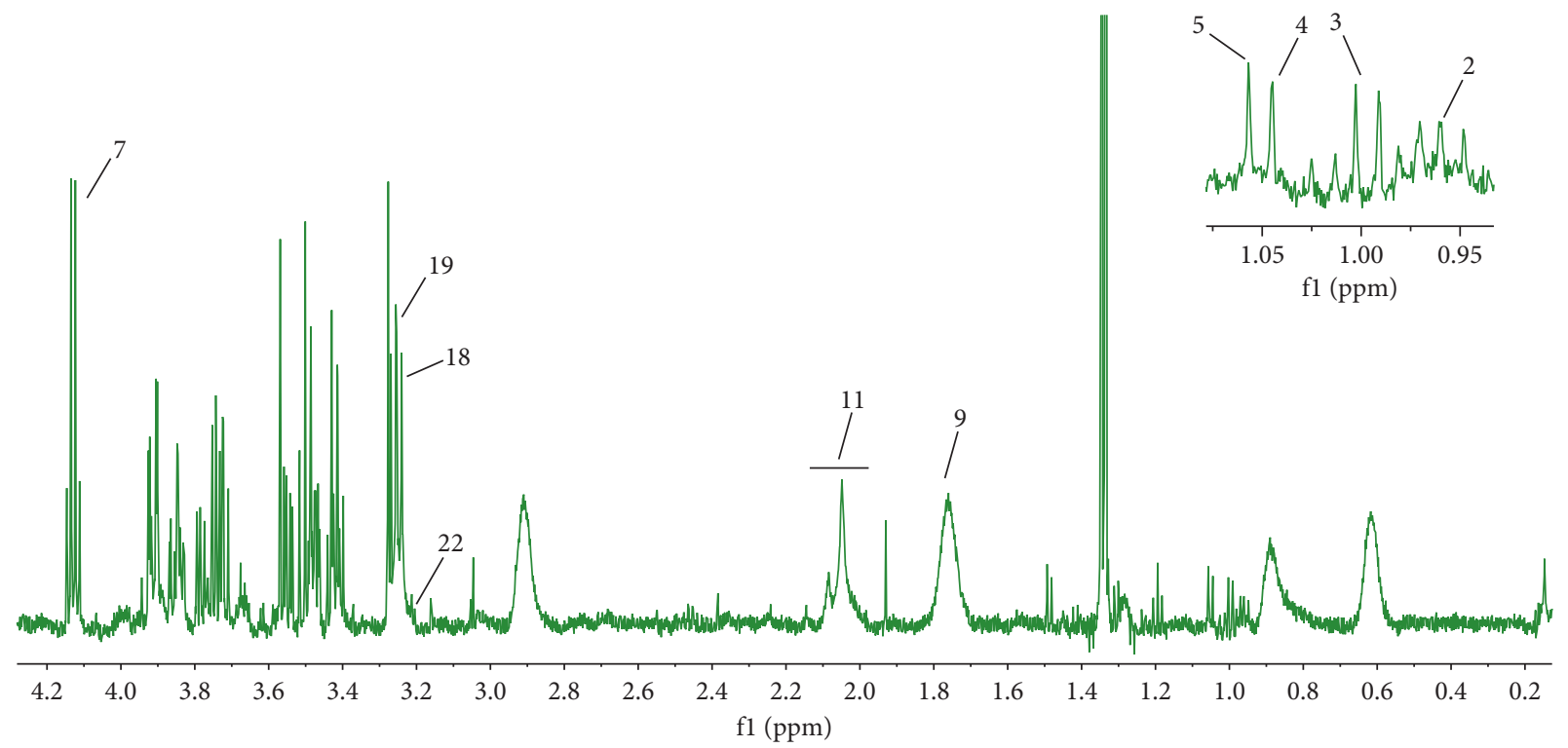

(c)

Figure 7: Representative ${ }^{1} \mathrm{H}-\mathrm{NMR}$ spectra from rabbits: (a) the NC group, (b) the AD group, and (c) the GXN group. (1) LDL, (2) leucine, (3) isoleucine, (4) valine, (5) isobutyric acid, (6) 3-hydroxybutyrate, (7) lactate, (8) alanine, (9) lysine, (10) Acetic acid, (11) glutamate, (12) pyruvate, (13) glutamine, (14) citric acid, (15) TMA, (16) creatine, (17) creatinine, (18) TMAO, (19) betaine, (20) proline, (21) glycerol, (22) L-carnitine, (23) glycine, and (24) glucose.

them with the NMR data available in the literature and the Human Metabolome Database (HMDB, http://www.hmdb. $\mathrm{ca} /$ ) [10]. The typical ${ }^{1} \mathrm{H}-\mathrm{NMR}$ spectrum of each group and metabolites identified are shown in Figure 7.

Multivariate statistical analysis and visualization were performed to more visually observe the changes in metabolites in $\mathrm{AD}$ rabbits and the improvement effect of administration of GXN. The PLS-DA score plot (Figure $8(\mathrm{a}) ; R^{2} X=0.915, R^{2} Y=0.832$, and $Q^{2}=0.619$ ) showed that the three groups were separated, indicating that the metabolites in the AD rabbits were disordered after modeling and that the GXN intervention regulated the metabolites in AD rabbits. Then, a permutation test ( $n=200)$ was subsequently conducted to validate the PLSDA and avoid overfitting. As shown in Figure 8(b), the permutation test suggested that the PLS-DA model had appropriate $R^{2} Y(0,0.44)$ and $\mathrm{Q}^{2}(0,-0.249)$ values, suggesting that the PLS-DA model was not overfitted and had good prediction ability.

Furthermore, OPLS-DA was conducted between the AD and GXN groups to determine the differentially altered metabolites. Consistent with the results of the PLS-DA score plot, the OPLS-DA model (Figure 8(c); $R^{2} X=0.787$, $R^{2} Y=0.994$, and $\left.Q^{2}=0.953\right)$ also suggested a separation between the AD and GXN groups. Furthermore, based on the $P$ values $(P<0.05$; ANOVA) and VIP values (VIP $>1$ ), 12 differentially altered metabolites were screened (Figure $8(\mathrm{~d})$ ). Compared with the AD group, the serum LDL, glycerol, glycine, TMAO, and creatine levels in the GXN group were significantly decreased $(P<0.05$ and $P<0.01)$, while leucine, isobutyric acid, valine, glutamate, 3-hydroxybutyrate, acetic acid, and citric acid levels were significantly increased $(P<0.05$ and $P<0.01)$, suggesting that GXN may ameliorate $\mathrm{AD}$ by regulating the levels of these metabolites. Finally, the 12 differentially altered metabolites mentioned above were imported into MetaboAnalyst 5.0 software (https://www.metaboanalyst.ca/) for a pathway enrichment analysis to identify the key metabolic pathways related to the effect of GXN on ameliorating AD. Seven metabolic pathways (whose $P<0.05$ or Impact $>0.1$ ) related to the effect of GXN on ameliorating AD were obtained (Figure 8(e)).

3.5. GXN Improved Neuronal Metabolism Activities in AD Rabbits. ${ }^{1} \mathrm{H}$-MRS was performed to evaluate the alterations of neuronal metabolism activity in the rabbit brain, and the metabolites from ${ }^{1} \mathrm{H}$-MRS spectra were identified in Figure 3(b). Quantitatively, in the AD group, the NAA/Cr and $\mathrm{Glx} / \mathrm{Cr}$ levels in the brain were significantly reduced compared to those in the NC group $(P<0.01$; Figure $3(\mathrm{c}))$, whereas the $\mathrm{Cho} / \mathrm{Cr}$ and $\mathrm{mI} / \mathrm{Cr}$ levels were significantly increased $(P<0.01)$. In contrast, GXN treatment produced $19.1 \%$ and $31.0 \%$ increases in the NAA/Cr and Glx/Cr levels, respectively $(P<0.05)$, and $31.3 \%$ and $18.3 \%$ reductions in the $\mathrm{Cho} / \mathrm{Cr}$ and $\mathrm{mI} / \mathrm{Cr}$ levels, respectively $(P<0.05$ and $P<0.01)$.

3.6. GXN Inhibited Apoptosis-Related Protein Expression and Enhanced Antioxidant Capacities in AD Rabbits. The expression of the Bax and Bcl-2 proteins was observed in the immunohistochemistry images. The nucleus was stained blue, and the positive staining was brown (Figures 9(a) and $9(c))$. In the GXN group, Bcl-2 expression in the cortex and hippocampus was remarkably increased $(P<0.05)$, while 


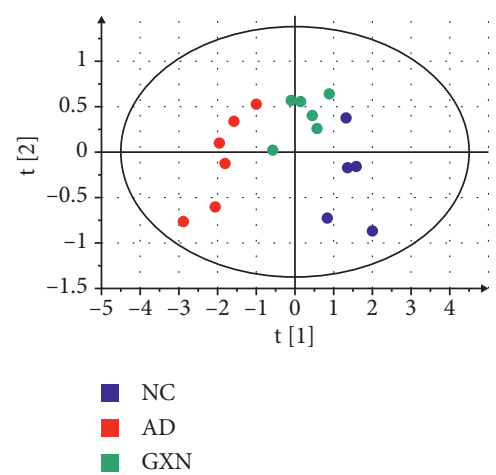

(a)

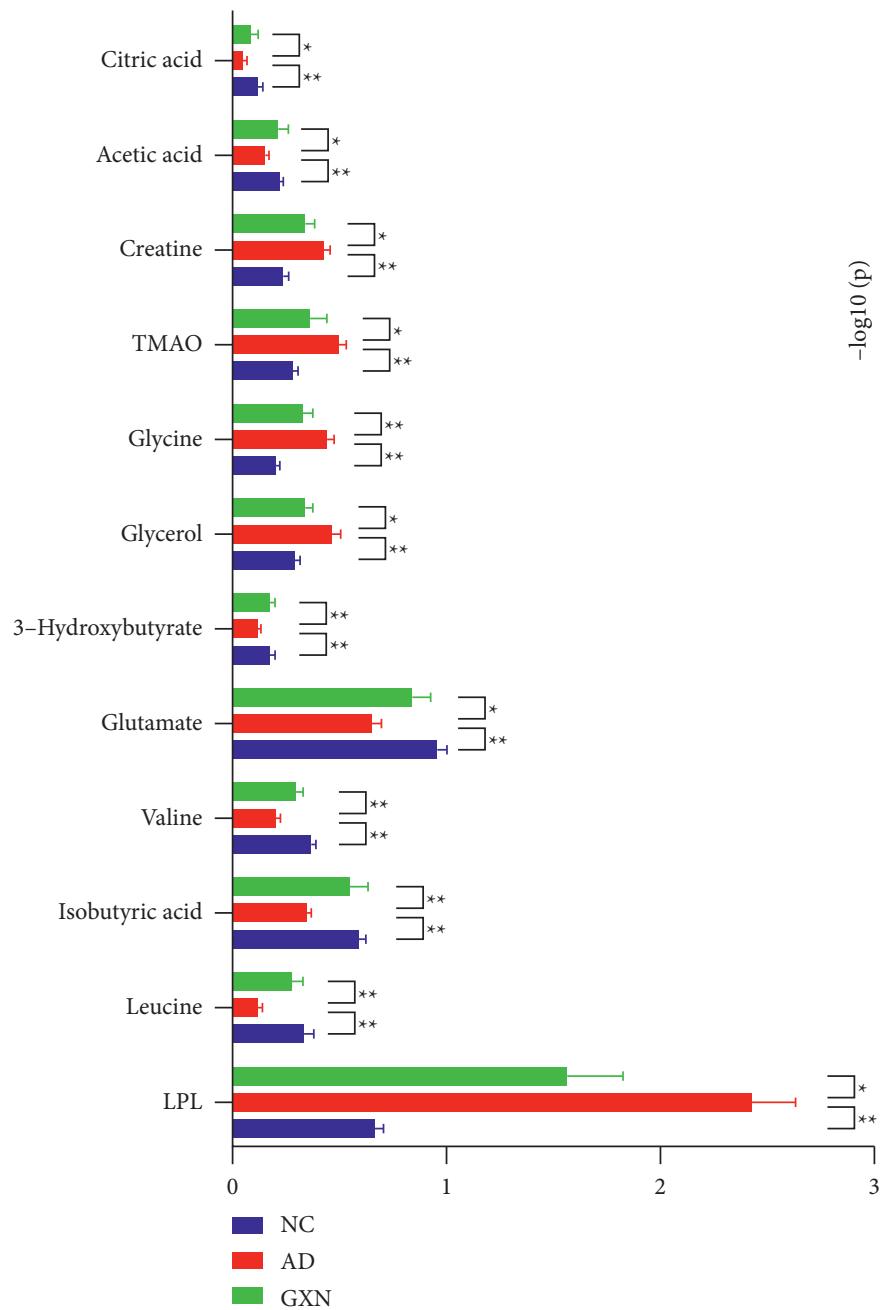

(d)

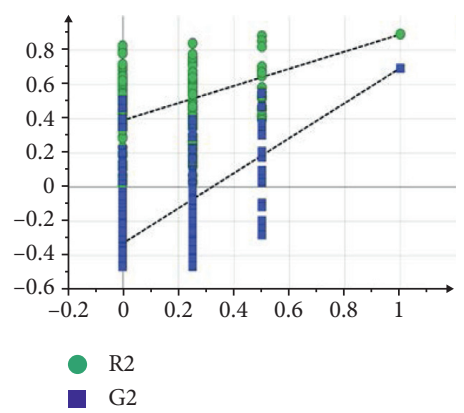

(b)

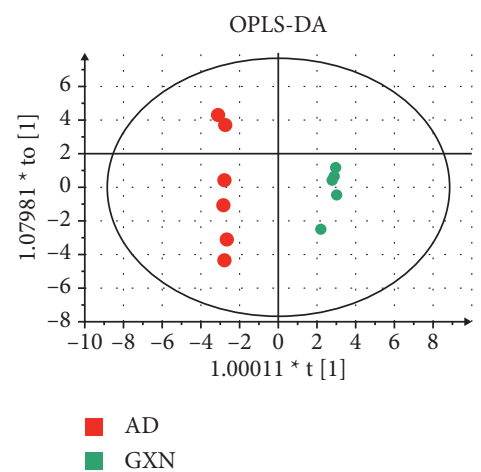

(c)

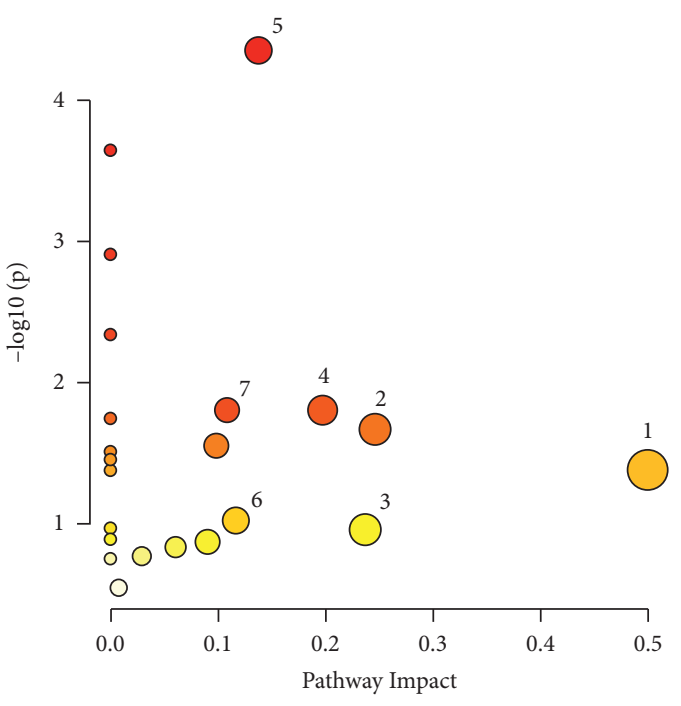

(e)

FIGURE 8: GXN altered serum metabolite levels in AD rabbits: (a) PLS-DA score plot for each group, (b) permutation test of the PLS-DA model, (c) OPLS-DA score plot of the AD group versus the GXN group, (d) relative serum contents of different metabolites in rabbits from each group, and (e) schematic representation of pathways identified by MetaboAnalyst 5.0. The ordinate indicates the significance level of the metabolic pathway, and the abscissa indicates the importance of the metabolic pathway. (1) D-glutamine and D-glutamate metabolism; 2. glycine, serine, and threonine metabolism; 3. glycerolipid metabolism; 4. alanine, aspartate, and glutamate metabolism; 5. glyoxylate and dicarboxylate metabolism; 6 . arginine biosynthesis; and 7. glutathione metabolism. $n=6$. Red and blue indicate positive correlations and negative correlations, respectively. 


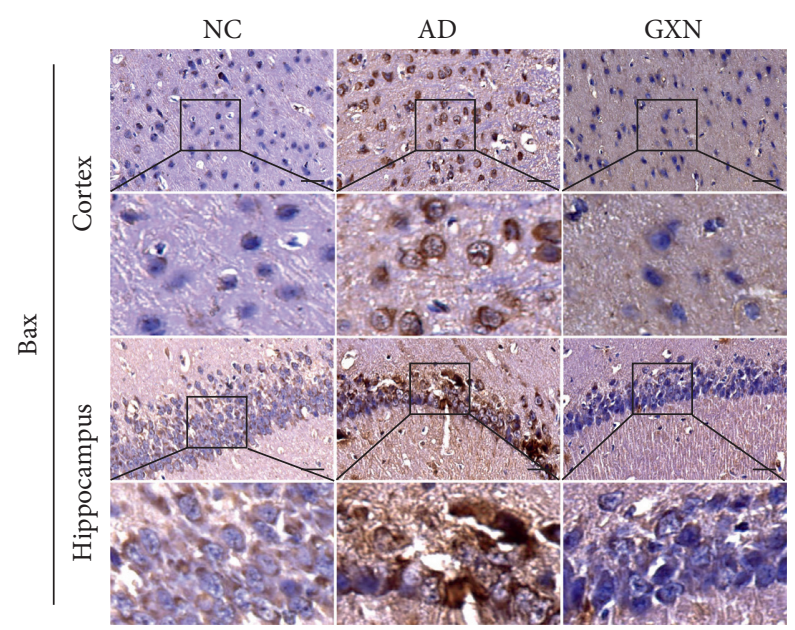

(a)

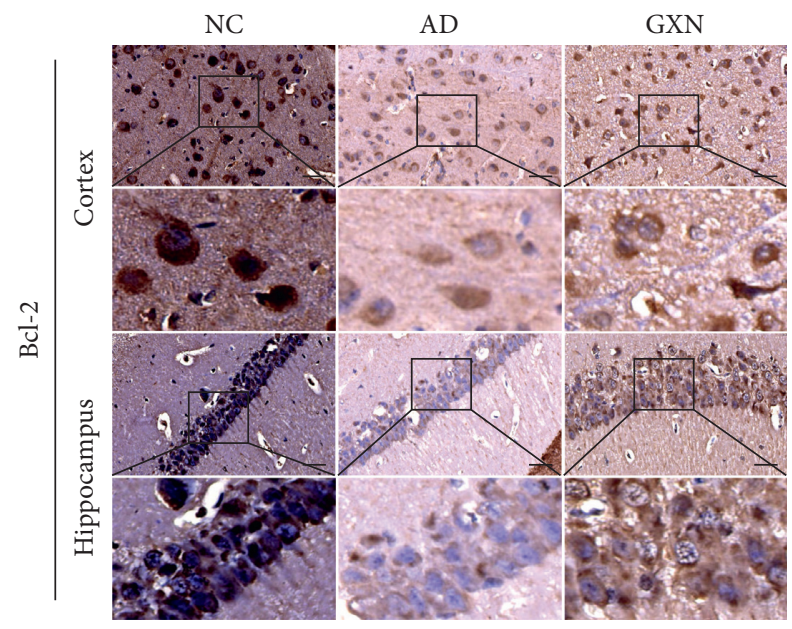

(c)

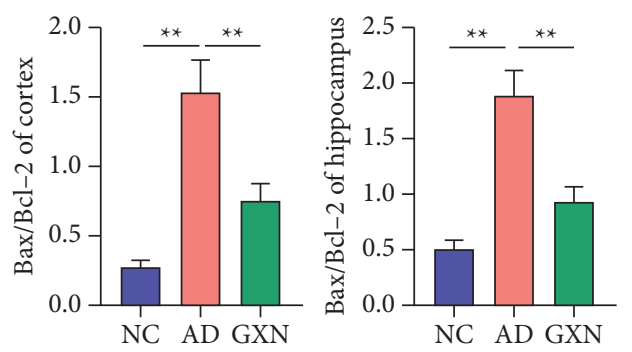

(e)
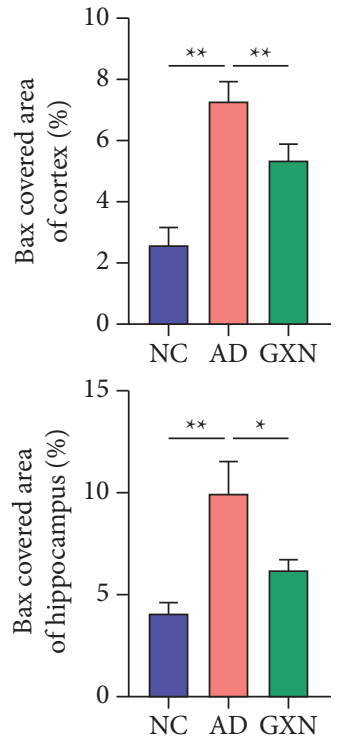

(b)
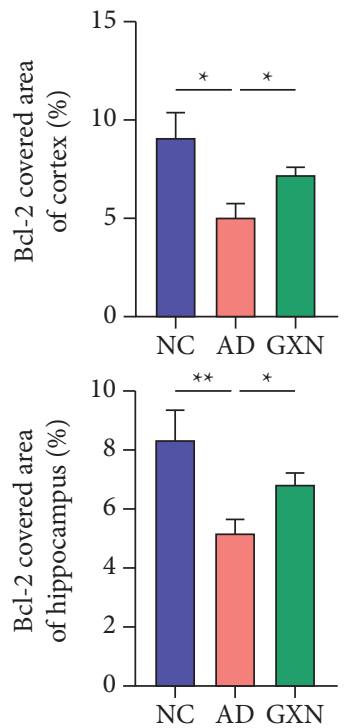

(d)

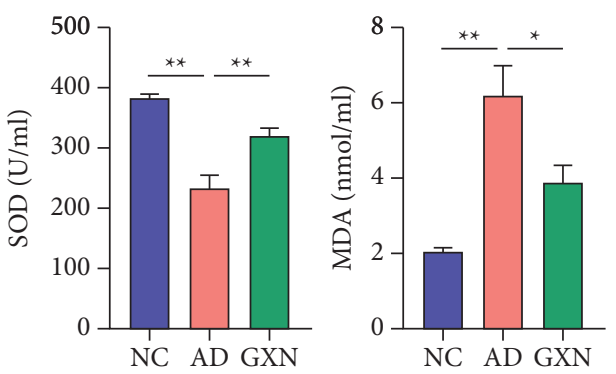

(f)

FIGURE 9: GXN inhibited apoptosis-related protein expression and enhanced serum antioxidant capacities. Representative images of Bax (a) and Bcl-2 staining (c) in three groups of rabbits. Scale bar $=50 \mathrm{~mm}$. Black boxes indicate the areas shown at higher magnification. Quantitative analysis of percentages of Bax-positive staining (b) and Bcl-2-positive staining (d) in each group. (e) Bax/Bcl-2 ratio in each group. (f) Changes in serum SOD and MDA levels in each group. $n=6$. 
Bax expression was remarkably decreased relative to that in the AD group $(P<0.05$ and $P<0.01$; Figures 9 (b) and $9(d))$. Furthermore, in the GXN group, the Bax to Bcl-2 ratio in the brain was significantly decreased compared to that in the AD group $(P<0.01$; Figure $9(\mathrm{e}))$. In addition, the GXN intervention remarkably increased SOD levels $(P<0.01)$ and significantly reduced MDA levels $(P<0.05$; Figure $9(f))$.

3.7. GXN Attenuated Neuronal Apoptosis in AD Rabbits. A TUNEL assay was conducted to observe neuronal apoptosis in rabbits. As shown in Figure 10, the AI of the cortex and hippocampus was significantly increased in the $\mathrm{AD}$ group compared to the NC group $(P<0.05)$, whereas the GXN intervention significantly decreased the AI of the cortex and hippocampus $(P<0.01)$.

\section{Discussion}

Our study confirmed that a high-cholesterol diet induced some typical $\mathrm{AD}$ symptoms and $\mathrm{AD}$ pathological manifestations in a rabbit model. In contrast, GXN treatment decreased the TC and LDL levels, the Firmicutes/Bacteroidetes ratio, and the abundance of $d g A-11$ gut_group; increased the abundance of Akkermansia; and improved serum and brain metabolite disorders. Meanwhile, GXN also increased SOD and Bcl-2 levels, decreased MDA and Bax levels, and ameliorated $\mathrm{A} \beta$ deposition, neuronal apoptosis, and memory deficits in $\mathrm{AD}$ rabbits induced by a highcholesterol diet. Based on our results, GXN attenuated AD.

Hypercholesterolemia is a significant risk factor for AD and was proven to enhance the activity of the $\beta$-secretase pathway that processes amyloid precursor protein through the formation of lipid rafts, thereby promoting $\mathrm{A} \beta$ deposition, aggravating $\mathrm{A} \beta$ neurotoxicity, and subsequently leading to the occurrence of $\mathrm{AD}$ [26]. In addition, previous studies have suggested that hypercholesterolemia is closely related to cognitive decline [27]. Clinically, patients with AD are characterized by progressive memory and cognitive decline [28]. These characteristics are consistent with the changes in the AD model rabbits fed a $2 \%$ cholesterol diet. In contrast, the GXN intervention significantly reduced TC and LDL-C levels, inhibited brain $\mathrm{A} \beta$ deposition, and improved memory and behaviors in AD rabbits. Thus, GXN may inhibit $\mathrm{A} \beta$ deposition and memory and behavioral deficits by reducing cholesterol levels in $\mathrm{AD}$ rabbits.

Based on accumulating evidence, the gut microbiota and its metabolites affect $\mathrm{AD}$; consequently, the gut microbiota is also considered a potential therapeutic target for $\mathrm{AD}$ $[29,30]$. AB-PAS staining evaluates the integrity of colonic epithelial barrier function [31]. Goblet cells secrete mucin, which covers the surface of the colonic mucosa to form a mucus barrier and maintain the integrity of the colonic barrier [32]. In addition, $16 \mathrm{~S}$ rRNA sequencing evaluates the changes in the gut microbiota structure to further reveal other potential mechanisms by which GXN ameliorates AD. Previous studies have found that a high-cholesterol diet causes gut microbiota dysbiosis, damage to colonic barrier integrity, and host metabolic disorders [18, 33]. Similarly, changes in gut microbiota diversity and a decreased number of mucus-secreting goblet cells per colonic crypt were observed in our study. In contrast, GXN significantly ameliorated gut microbiota diversity and increased the number of goblet cells, suggesting that GXN may exert its effect by improving the gut microbiota and colon barrier permeability. In addition, GXN remarkably increased the abundance of Akkermansia and decreased the abundance of the dgA-11_gut_group in $\mathrm{AD}$ rabbits. Akkermansia is a representative genus in the human gut microbiota in the Verrucomicrobia phylum, and a high-fat diet can reduce its abundance [34]. As previously reported, Akkermansia preserves colonic barrier permeability, reduces blood endotoxin levels, improves insulin resistance, and protects $\mathrm{AD}$ model mice from cognitive deficits and amyloid pathology, indicating that it may slow the progression of $\mathrm{AD}$ [35]. In addition, as a bacterium that produces short-chain fatty acids (SCFAs) [36], a decrease in the level of Akkermansia was also considered to cause inflammation, impaired glucose tolerance, and diet-induced obesity [37], thereby participating in the development of $\mathrm{AD}$. In addition, the $d g A$ 11_gut_group was proposed to be related to lipid metabolism disorders, which are vital factors contributing to AD [38]. Furthermore, the LEfSe analysis confirmed that Akkermansia and the dgA-11_gut_group might be the target bacteria of the anti-AD effects of GXN, and a subsequent correlation analysis showed that Akkermansia and the $d g A$ 11_gut_group were significantly correlated with AD-related clinical indices. Therefore, these results indicated that GXN may exert an anti-AD effect by regulating Akkermansia and the $d g A-11$ gut_group and ameliorating gut microbiota dysbiosis.

${ }^{1} \mathrm{H}-\mathrm{NMR}$ metabolomics and pathway enrichment analyses showed that the GXN intervention significantly reduced LDL and TMAO levels in AD rabbits, increased glutamate, citric acid, 3-hydroxybutyric, and leucine levels and was involved in D-glutamine and D-glutamate metabolism and glutathione metabolism. Glutamate, a precursor for the synthesis of the natural antioxidant glutathione (GSH), is presumed to play a vital role in cellular antioxidant defenses [39]. GSH prevents oxidative damage and neuronal apoptosis by reducing $\mathrm{H}_{2} \mathrm{O}_{2}$ levels, thereby delaying the progression of $\mathrm{AD}$ [39]. In addition, TMAO (a gut microbiota-related metabolite) levels are significantly increased in patients with AD and positively correlate with $\mathrm{AD}$ markers (A $\beta$ and $\mathrm{p}$-tau) [40]. Citric acid is a significant intermediate product of the tricarboxylic acid cycle and the hub of the metabolic connection of lipids [41]. Leucine and 3-hydroxybutyric acid affect lipid metabolism and synthesis and intrahepatic mitochondrial lipid metabolism [42, 43]. Thus, GXN may ameliorate AD by inhibiting lipid synthesis and reducing lipid peroxidation and neuronal apoptosis.

Furthermore, ${ }^{1} \mathrm{H}$-MRS indicated that GXN significantly enhanced neuronal metabolism activities, such as increasing Glx/Cr and NAA/Cr levels and decreasing Cho/Cr and mI/Cr levels. Studies have shown that metabolic changes in the brain are significantly related to cognitive decline, and the symptoms of dementia in patients with $\mathrm{AD}$ are postulated to be associated with a decline in neuronal metabolic activities 


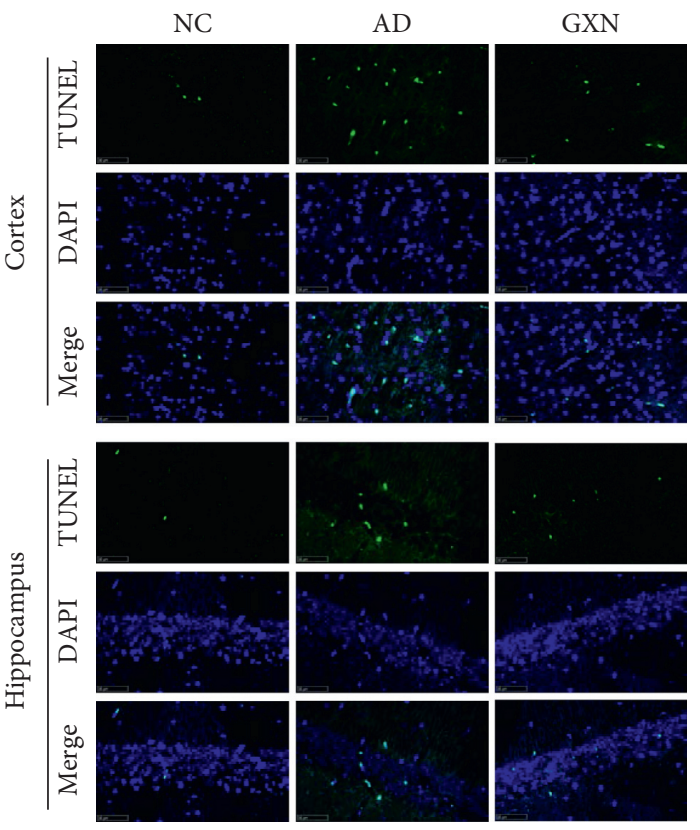

(a)
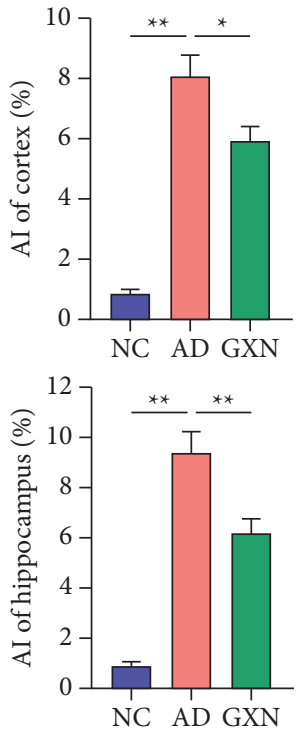

(b)

FIGURE 10: GXN attenuated neuronal apoptosis in AD rabbits. (a) Representative images of TUNEL staining in the three groups of rabbits. Scale bar $=50 \mathrm{~mm}$. (b) Quantitative analysis of the percentage of TUNEL-positive cells in each group. $n=6$.

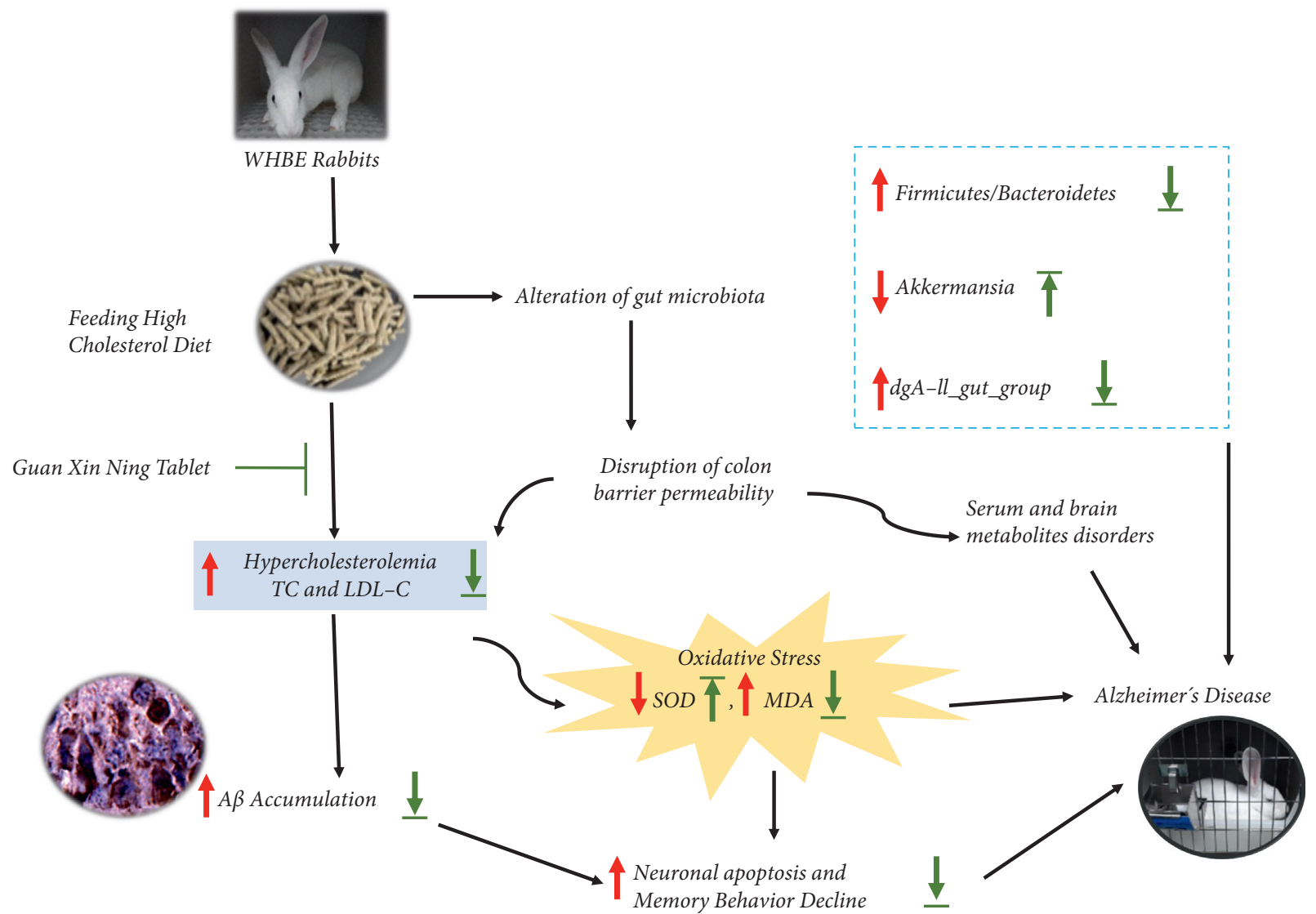

FIGURE 11: Schematic diagram of possible mechanisms by which GXN ameliorates AD in high-cholesterol diet-induced rabbits. GXN reduces cholesterol levels, the Firmicutes/Bacteroidetes ratio, and the abundance of $d g A-11$ gut_group; increases the abundance of Akkermansia; and attenuates serum and brain metabolite disorders. Meanwhile, GXN also increases SOD levels, decreases MDA levels, and attenuates $\mathrm{A} \beta$ deposition and neuronal apoptosis, thereby ameliorating $\mathrm{AD}$ symptoms in rabbits fed a high-cholesterol diet. Arrows indicate statistically significant increases and decreases $(P<0.05)$. The red and green arrows suggest the changes in AD model rabbits and the therapeutic effects of GXN, respectively. 
$[8,9]$. Therefore, an abnormal metabolic function in the brain is considered a critical pathological factor that causes AD [44]. NAA is a specific biomarker of the severity of the neuronal injury, and its level is significantly decreased in patients with $\mathrm{AD}$ [45]. Glx is the most abundant excitatory neurotransmitter in the brain and has multiple functions in the nervous system [46]. Decreased levels of Glx reflect the loss or decreased activity of glutamatergic neurons, which is a significant pathological feature in the brains of patients with AD. In addition, Cho is one of the components of cell membrane phospholipid metabolism and is involved in the synthesis and metabolism of cell membranes [47]. mI participates in astrocyte activation and proliferation and is a significant marker indicating the severity of inflammation in the brain [48]. Thus, GXN may stabilize nerve cell membranes, inhibit the activation of glial cells, and improve neuronal metabolism in $\mathrm{AD}$ rabbits, thereby ameliorating $\mathrm{AD}$.

In addition, under normal physiological conditions, reactive oxygen species (ROS) are eliminated by the antioxidant substances in the body [49]. Hypercholesterolemia, gut microbiota dysbiosis, and destruction of the colon barrier are related to oxidative stress $[50,51]$. In individuals with $\mathrm{AD}, \mathrm{A} \beta$ neurotoxicity and oxidative stress damage neurons by inducing apoptosis $[26,52]$, thereby leading to cognitive decline [53]. In our study, the GXN intervention significantly reduced the $\mathrm{A} \beta$ load in $\mathrm{AD}$ rabbits. Furthermore, four markers were examined to evaluate oxidative stress and the changes in apoptotic activities: SOD (a free radical scavenger used to evaluate the generation of free radicals), MDA (a sensitive indicator of the strength of oxidative stress), and Bax and Bcl2 (vital regulatory genes involved in cell apoptosis). Bax promotes apoptosis, while $\mathrm{Bcl}-2$ inhibits apoptosis. The Bax to $\mathrm{Bcl}-2$ ratio determines the degree of apoptosis: the higher the ratio, the greater the apoptosis. Our study suggested that GXN remarkably suppressed oxidative stress and apoptosis-related protein expression. Moreover, the TUNEL assay also indicated that the GXN intervention significantly ameliorated apoptosis in the cortex and hippocampus. Based on these results, GXN may have the potential to prevent neuronal apoptosis by suppressing $\mathrm{A} \beta$ neurotoxicity, oxidative stress, and Bax expression and promoting Bcl-2 expression.

However, our study had some limitations. First, although the reliability of the rabbit $\mathrm{AD}$ model and the effectiveness of drug interventions have been reported, the present study did not include a positive reference drug group, which may not fully reflect the advantages of GXN. Second, although the characteristic metabolite changes induced by GXN to ameliorate $\mathrm{AD}$ were obtained using ${ }^{1} \mathrm{H}-\mathrm{NMR}$, some other potential metabolites may be omitted due to the limited number of metabolites detected using this method. Therefore, the design of positive reference drugs and detection of metabolites using q-TOF/MS or Orbitrap-based methods are necessary to further evaluate the efficacy and mechanism of action of GXN in AD.

\section{Conclusion}

In conclusion, our current study suggested that GXN ameliorated AD symptoms by reducing cholesterol levels and $\mathrm{A} \beta$ deposition, regulating disorders of gut microbiota and serum metabolites, enhancing neuronal metabolic activities, and inhibiting oxidative stress and neuronal apoptosis (Figure 11). Together, these results reveal the possible mechanisms by which GXN ameliorates $\mathrm{AD}$, providing experimental evidence that GXN delays AD progression.

\section{Data Availability}

The data are available from the corresponding author on reasonable request.

\section{Ethical Approval}

All the manipulations comply with the Institutional Animal Care and Use Committee (IACUC) of Zhejiang Chinese Medical University (approval no. 20190930-06).

\section{Conflicts of Interest}

The authors declare that there are no conflicts of interest regarding the publication of this paper.

\section{Authors' Contributions}

YP and MC designed the experiment; FZ, YX, LS, JH, SX, JL, $\mathrm{ZS}$, JH, and YP conducted the experiment; FZ and MC performed the data analysis; and FZ and YP wrote the manuscript.

\section{Acknowledgments}

This research was funded by grants from the National Natural Science Foundation of China (No. 31970514), Opening Project of Key Laboratory of Integrative Chinese and Western Medicine for the Diagnosis and Treatment of Circulatory Diseases of Zhejiang Province (No. 2C32102), Zhejiang Basic Public Welfare Research Project (No. LGD19C040002), and Zhejiang College Students' Innovation and Entrepreneurship Training Program (S202010344036).

\section{References}

[1] R. Brito-Aguilar, "Dementia around the world and the Latin America and Mexican scenarios," Journal of Alzheimer's Disease, vol. 71, no. 1, pp. 1-5, 2019.

[2] S. E. Lacher, A. Alazizi, X. Wang et al., "A hypermorphic antioxidant response element is associated with increased MS4A6A expression and Alzheimer's disease," Redox biology, vol. 14, pp. 686-693, 2018.

[3] J. Cummings, G. Lee, A. Ritter, M. Sabbagh, and K. Zhong, "Alzheimer's disease drug development pipeline: 2020," Alzheimer's \& Dementia, vol. 6, Article ID e12050, 2020.

[4] T. R. Ferreira, L. C. Lopes, and C. d. C. Bergamaschi, "Frequency and severity of adverse drug reactions to medications prescribed for Alzheimer's disease in a Brazilian city: crosssectional study," Frontiers in Pharmacology, vol. 11, Article ID 538095, 2020.

[5] S.-M. Fereshtehnejad, K. Johnell, and M. Eriksdotter, "Antidementia drugs and Co-medication among patients with Alzheimer's disease," Drugs \& Aging, vol. 31, no. 3, pp. 215-224, 2014. 
[6] L. J. Spielman, D. L. Gibson, and A. Klegeris, "Unhealthy gut, unhealthy brain: the role of the intestinal microbiota in neurodegenerative diseases," Neurochemistry International, vol. 120, pp. 149-163, 2018.

[7] W. Jia, C. Rajani, R. Kaddurah-Daouk, and H. Li, "Expert insights: the potential role of the gut microbiome-bile acidbrain axis in the development and progression of Alzheimer's disease and hepatic encephalopathy," Medicinal Research Reviews, vol. 40, no. 4, pp. 1496-1507, 2020.

[8] L. Chang, S. M. Munsaka, S. Kraft-Terry, and T. Ernst, "Magnetic resonance spectroscopy to assess neuroinflammation and neuropathic pain," Journal of Neuroimmune Pharmacology, vol. 8, no. 3, pp. 576-593, 2013.

[9] K. Kantarci, "Proton MRS in mild cognitive impairment," Journal of Magnetic Resonance Imaging, vol. 37, no. 4, pp. 770-777, 2013.

[10] A. Maimaiti, Y. Tao, W. Minmin et al., "Dracocephalum moldavica improvement of total flavonoids from L. In rats with chronic mountain sickness through H-NMR metabonomics," Evidence-Based Complementary and Alternative Medicine: eCAM, vol. 2021, Article ID 6695346, 13 pages, 2021.

[11] J. Lu, P. Guo, X. Liu et al., "Herbal formula fo shou san attenuates Alzheimer's disease-related pathologies via the gutliver-brain Axis in APP/PS1 mouse model of Alzheimer's disease," Evidence-Based Complementary and Alternative Medicine: eCAM, vol. 2019, Article ID 8302950, 14 pages, 2019.

[12] Y. Liu, T. Du, W. Zhang et al., " $\beta$ Modified huang-lian-jie-du decoction ameliorates A synaptotoxicity in a murine model of Alzheimer's disease," Oxidative Medicine and Cellular Longevity, vol. 2019, Article ID 8340192, 27 pages, 2019.

[13] M.-L. Wang, Q.-Q. Yang, X.-H. Ying et al., "Network pharmacology-based approach uncovers the mechanism of GuanXinNing tablet for treating thrombus by MAPKs signal pathway," Frontiers in Pharmacology, vol. 11, p. 652, 2020.

[14] X. Zhou, G. Cui, H. H. Tseng et al., "Vascular contributions to cognitive impairment and treatments with traditional Chinese medicine," Evidence-based Complementary and Alternative Medicine: eCAM, vol. 2016, Article ID 9627258, 12 pages, 2016.

[15] M. Wang, M. Yao, J. Liu et al., "Ligusticum chuanxiong exerts neuroprotection by promoting adult neurogenesis and inhibiting inflammation in the hippocampus of ME cerebral ischemia rats," Journal of Ethnopharmacology, vol. 249, Article ID 112385, 2020.

[16] Y. Song, M.-s. Wu, G. Tao, M.-w. Lu, J. Lin, and J.-q. Huang, "Feruloylated oligosaccharides and ferulic acid alter gut microbiome to alleviate diabetic syndrome," Food Research International, vol. 137, Article ID 109410, 2020.

[17] L. Li, R. Li, R. Zhu et al., "Salvianolic acid B prevents body weight gain and regulates gut microbiota and LPS/TLR4 signaling pathway in high-fat diet-induced obese mice," Food \& Function, vol. 11, no. 10, pp. 8743-8756, 2020.

[18] H. Cui, Y. Li, Y. Wang et al., "Da-chai-hu decoction ameliorates high fat diet-induced nonalcoholic fatty liver disease through remodeling the gut microbiota and modulating the serum metabolism," Frontiers in Pharmacology, vol. 11, Article ID 584090, 2020.

[19] D. L. Sparks and B. G. Schreurs, "Trace amounts of copper in water induce-amyloid plaques and learning deficits in a rabbit model of Alzheimer's disease," Proceedings of the National Academy of Sciences, vol. 100, no. 19, pp. 11065-11069, 2003.
[20] E. M. Johnstone, M. O. Chaney, F. H. Norris, R. Pascual, and S. P. Little, "Conservation of the sequence of the Alzheimer's disease amyloid peptide in dog, polar bear and five other mammals by cross-species polymerase chain reaction analysis," Molecular Brain Research, vol. 10, no. 4, pp. 299-305, 1991.

[21] Q. Y. Liu, M. N. Chang, J. X. Lei et al., "Identification of microRNAs involved in Alzheimer's progression using a rabbit model of the disease," American journal of neurodegenerative disease, vol. 3, pp. 33-44, 2014.

[22] Y. Pan, J. Xu, C. Chen et al., "Royal jelly reduces cholesterol levels, ameliorates $\mathrm{A} \beta$ pathology and enhances neuronal metabolic activities in a rabbit model of Alzheimer's disease," Frontiers in Aging Neuroscience, vol. 10, p. 50, 2018.

[23] Y. Pan, J. Xu, P. Jin et al., "Royal jelly ameliorates behavioral deficits, cholinergic system deficiency, and autonomic nervous dysfunction in ovariectomized cholesterol-fed rabbits," Molecules, vol. 24, 2019.

[24] P. Jin, Y. Pan, Z. Pan et al., "Correction to: Alzheimer-like brain metabolic and structural features in cholesterol-fed rabbit detected by magnetic resonance imaging," Lipids in Health and Disease, vol. 17, no. 1, p. 204, 2018.

[25] X. Hao, P. He, Y. Ni et al., "Escherichia coli effect of xueniao capsule on-induced acute pyelonephritis rats by H NMRbased metabolomic approach," Evidence-Based Complementary And Alternative Medicine: eCAM, vol. 2019, Article ID 6723956, 12 pages, 2019.

[26] C. Marquer, V. Devauges, J. C. Cossec et al., "Local cholesterol increase triggers amyloid precursor protein-Bacel clustering in lipid rafts and rapid endocytosis," The FASEB Journal, vol. 25, no. 4, pp. 1295-1305, 2011.

[27] S. R. Cox, D. M. Lyall, S. J. Ritchie et al., "Associations between vascular risk factors and brain MRI indices in UK Biobank," European Heart Journal, vol. 40, no. 28, pp. 2290-2300, 2019.

[28] T. Saari, I. Hallikainen, T. Hintsa, and A. M. Koivisto, "Neuropsychiatric symptoms and activities of daily living in Alzheimer's disease: ALSOVA 5-year follow-up study," International Psychogeriatrics, vol. 32, no. 6, pp. 741-751, 2020.

[29] F. Panza, M. Lozupone, V. Solfrizzi, M. Watling, and B. P. Imbimbo, "Time to test antibacterial therapy in Alzheimer's disease," Brain: A Journal of Neurology, vol. 142, pp. 2905-2929, 2019.

[30] P. Liu, L. Wu, G. Peng et al., "Altered microbiomes distinguish Alzheimer's disease from amnestic mild cognitive impairment and health in a Chinese cohort," Brain, Behavior, and Immunity, vol. 80, pp. 633-643, 2019.

[31] I. Röhe, F. J. Hüttner, J. Plendl, B. Drewes, and J. Zentek, "Comparison of different histological protocols for the preservation and quantification of the intestinal mucus layer in pigs," European Journal of Histochemistry, vol. 62, p. 2874, 2018.

[32] P. Paone and P. D. Cani, "Mucus barrier, mucins and gut microbiota: the expected slimy partners?" Gut, vol. 69 , no. 12 , pp. 2232-2243, 2020.

[33] J.-C. Lee, H.-Y. Lee, T. K. Kim et al., "Obesogenic diet-induced gut barrier dysfunction and pathobiont expansion aggravate experimental colitis," PLoS One, vol. 12, no. 11, Article ID e0187515, 2017.

[34] J. Wu, Y. Xu, J. Su et al., "Roles of gut microbiota and metabolites in a homogalacturonan-type pectic polysaccharide from Ficus pumila Linn. fruits mediated amelioration of obesity," Carbohydrate Polymers, vol. 248, Article ID 116780, 2020. 
[35] Z. Ou, L. Deng, Z. Lu et al., "Protective effects of Akkermansia muciniphila on cognitive deficits and amyloid pathology in a mouse model of Alzheimer's disease," Nutrition \& Diabetes, vol. 10 , no. 1 , p. $12,2020$.

[36] P. Van den Abbeele, P. Gérard, S. Rabot et al., "Arabinoxylans and inulin differentially modulate the mucosal and luminal gut microbiota and mucin-degradation in humanized rats," Environmental Microbiology, vol. 13, no. 10, pp. 2667-2680, 2011.

[37] M. Bellahcene, J. F. O’Dowd, E. T. Wargent et al., "Male mice that lack the G-protein-coupled receptor GPR41 have low energy expenditure and increased body fat content," British Journal of Nutrition, vol. 109, no. 10, pp. 1755-1764, 2013.

[38] X. Sun, J. Shen, C. Liu et al., "L-arginine and N-carbamoylglutamic acid supplementation enhance young rabbit growth and immunity by regulating intestinal microbial community," Asian-Australasian Journal of Animal Sciences, vol. 30, no. 1, pp. 166-176, 2019.

[39] T. W. Sedlak, B. D. Paul, G. M. Parker et al., "The glutathione cycle shapes synaptic glutamate activity," Proceedings of the National Academy of Sciences, vol. 116, no. 7, pp. 2701-2706, 2019.

[40] N. M. Vogt, K. A. Romano, B. F. Darst et al., "The gut microbiota-derived metabolite trimethylamine $\mathrm{N}$-oxide is elevated in Alzheimer's disease," Alzheimer's Research \& Therapy, vol. 10, no. 1, p. 124, 2018.

[41] M. Akram, "Citric acid cycle and role of its intermediates in metabolism," Cell Biochemistry and Biophysics, vol. 68, no. 3, pp. 475-478, 2014.

[42] N. Møller, "Ketone body, 3-hydroxybutyrate: minor metabolite - major medical manifestations," Journal of Clinical Endocrinology \& Metabolism, vol. 105, 2020.

[43] Q. Ma, X. Zhou, L. Hu, J. Chen, J. Zhu, and A. Shan, "Leucine and isoleucine have similar effects on reducing lipid accumulation, improving insulin sensitivity and increasing the browning of WAT in high-fat diet-induced obese mice," Food \& Function, vol. 11, no. 3, pp. 2279-2290, 2020.

[44] S. Liang, J. Huang, W. Liu et al., "Magnetic resonance spectroscopy analysis of neurochemical changes in the atrophic hippocampus of APP/PS1 transgenic mice," Behavioural Brain Research, vol. 335, pp. 26-31, 2017.

[45] H. R. Griffith, J. A. Hollander, O. C. Okonkwo, T. O’Brien, R. L. Watts, and D. C. Marson, "Brain metabolism differs in Alzheimer's disease and Parkinson's disease dementia," Alzheimer's and Dementia, vol. 4, no. 6, pp. 421-427, 2008.

[46] J. Penner, R. Rupsingh, M. Smith, J. L. Wells, M. J. Borrie, and R. Bartha, "Increased glutamate in the hippocampus after galantamine treatment for Alzheimer disease," Progress in Neuro-Psychopharmacology and Biological Psychiatry, vol. 34, no. 1, pp. 104-110, 2010.

[47] N. Zhang, X. Song, R. Bartha et al., "Advances in high-field magnetic resonance spectroscopy in Alzheimer's disease," Current Alzheimer Research, vol. 11, no. 4, pp. 367-388, 2014.

[48] C.-M. Moon, B.-C. Kim, and G.-W. Jeong, "Effects of donepezil on brain morphometric and metabolic changes in patients with Alzheimer's disease: a DARTEL-based VBM and 1H-MRS," Magnetic Resonance Imaging, vol. 34, no. 7, pp. 1008-1016, 2016.

[49] K. Wang, X. Jin, Q. Li et al., "Propolis from different geographic origins decreases intestinal inflammation and Bacteroides spp. populations in a model of DSS-induced colitis," Molecular Nutrition \& Food Research, vol. 62, Article ID e1800080, 2018.
[50] Y. Gao, Y. Liu, F. Ma et al., "Lactobacillus plantarum Y44 alleviates oxidative stress by regulating gut microbiota and colonic barrier function in Balb/C mice with subcutaneous d-galactose injection," Food \& Function, vol. 12, no. 1, pp. 373-386, 2021.

[51] K. Wang, Z. Wan, A. Ou et al., "Monofloral honey from a medical plant, Prunella Vulgaris, protected against dextran sulfate sodium-induced ulcerative colitis via modulating gut microbial populations in rats," Food \& Function, vol. 10, no. 7, pp. 3828-3838, 2019.

[52] P. Mecocci, U. MacGarvey, and M. F. Beal, "Oxidative damage to mitochondrial DNA is increased in Alzheimer's disease," Annals of Neurology, vol. 36, no. 5, pp. 747-751, 1994.

[53] J. P. Teixeira, A. A. de Castro, F. V. Soares, E. F. F. da Cunha, and T. C. Ramalho, "Future therapeutic perspectives into the Alzheimer's disease targeting the oxidative stress hypothesis," Molecules, vol. 24, 2019. 